\title{
Reconstructing the Deflector Images From the Ultrasonic Echo Signals by the Maximum Entropy Method
}

\author{
E. G Bazulin ${ }^{1}$ \\ ${ }^{1}$ Research and Production Center (ECHO+) LLC, Twardowski street 8, Moscow, Russia \\ Correspondence: E. G Bazulin, Research and Production Center (ECHO+) LLC, Twardowski street 8, Moscow, \\ Russia. E-mail: bazulin@echoplus.ru
}

\author{
Received: October 10, 2014 Accepted: October 27, 2014 Online Published: November 17, 2014 \\ doi:10.5539/apr.v6n6p109 URL: http://dx.doi.org/10.5539/apr.v6n6p109
}

\begin{abstract}
This article proposes using the maximum entropy method (MEM) for processing the ultrasonic echo signals in order to restore the deflectors images with high signal/noise ratio and small lateral petals of the point scattering function. When processing the ultrasonic echo signals, it is possible to take into consideration the pulses propagation paths, together with both their deflections from uneven boundary of inspected object and the respective wave type transformation. In model experiments the images of deflectors were received in two registering modes: in the combined mode - by using the conventional single element probe; in double and triple scan mode - by using the antenna array. The deflection from uneven surface during the echo signals acquisition had been taken into account in both modes. The restored images had a resolution exceeding the one required by Rayleigh criterion. MEM allows obtaining the defect images of low-level lateral petals by processing less than $10 \%$ of echo signals full set.
\end{abstract}

Keywords: automated ultrasonic nondestructive testing, dual scanning, Full Matrix Capture (FMC), triple scanning, antenna array, maximum entropy method (MEM), SAFT, C-SAFT, Total Focusing Method (TFM), digital focusing array (DFA)

\section{Introduction}

Getting the information on the internal structure of industrial facilities is an actual problem belonging to a class of problems inverse to that one of scattering, which is to determine the quantitative characteristics of unknown flaws on the basis of observing the scattered irradiating field. The most important problem of nondestructive testing is the found deflectors classifying and determining their sizes. This information may be used by experts on strength calculations to assess the operating life of the inspected objects.

In practice, coherent systems of ultrasonic nondestructive testing are being widely used. These systems register the echo signals by means of several single-plate probes moving over the surface of the inspected object. To restore the flaw images from the acquired echo signals, the algorithms based on the Born approximated solution of reverse scalar problem of scattering are normally used. Here are some of them: Synthetic Aperture Focusing Technique (SAFT) method (Hall, Doctor, Reid, Littlefield, \& Gilbert, 1987; Erhard, Lucht, Schulz, Montag, Wüstenberg, \& Beine, 2000; Osetrov, 1991), the method of angular spectrums (Goodman, 1968) and the method of Projection in the Spectral Space (PSS) (Mayer, Markelein, Langenberg, \& Kreutter, 1990; Badalyan \& Bazulin, 1988). Practical realization of the last method is especially effective in 3D-option due to high speed of getting the image. SAFT method provides images of alike quality. But in this case data processing takes place not in a spectral space with its fast Fourier transformation, but in time sphere and, therefore, takes more time. However, SAFT method enables to consider the multiple deflections of ultrasonic pulse from uneven boundary of the inspected object and, in the same time, the effect of wave type transformation, and thus has a very important advantage over PSS method, especially today that rapid development of computer engineering allows restoring deflector images with a frequency of more than $10 \mathrm{~Hz}$.

Recently the equipment exploiting the antenna arrays (AA), or matrixes, for emitting and receiving the ultrasound has become actively used in practice of ultrasonic testing. As a rule these devices operate in the mode of phased antenna arrays (PA) providing instrumental (hardware) focusing the ultrasound due to multichannel emitting and receiving the pulses with timed delays. However, PA-flaw detectors have a number of disadvantages (Bazulin, 2013), one of them being the defocusing and the shifting of deflectors glares from their actual locations proportionally to the distance from the focusing line. The increasing the antenna array or matrix aperture leads, on 
the one hand, to the improving of the image quality in the focusing area, and, on the other hand, to decreasing of dimensions of this very area. This problem is particularly acute in inspecting the thick-walled objects with help of 32-element PA (Braconnier, Okuda, \& Dao, 2009). A technology of dynamic depth focusing (DDF) (Olympus, 2007) was developed in order to partially eliminate such a negative effect.

One of the ways to obtain the deflectors images of higher quality is to initially receive the echo signals by antenna array operating in the dual scan mode (Bazulin, Golubev, \& Kokolev, 2009) in which the echo signals are being registrated by all pairs of array elements. In the article (Chatillon, Fidahoussen, Iakovleva, \& Calmon, 2009) such a mode of echo signals acquisition is called the Full Matrix Capture (FMC). As the dimensions of antenna matrix elements are commensurable with the wave length, each element forms a field of wide directional diagram (Danilov, Samokrutov, \& Lyutkevich, 2003) that enables acquisition of lots of pulses at longitudinal and transverse waves running from the deflector either directly or after they have been deflected by the borders of the inspected object. At the second stage the deflectors images are being restored by combined SAFT (C-SAFT) (Kovalev, Kozlov, Samokrutov, Shevaldykin, \& Yakovlev, 1990) method using the echo signals measured in dual scan mode. In article (Bazulin, 2011) this method, designated as TS-M-C-SAFT, is generalized for cases of multiple deflections from uneven boundary of the inspected object; the method also takes into account the transformation of wave type, it is applicable to the mode of triple scan when antenna array operating in the mode of dual scan moves along the inspected object. The C-SAFT method is also referred to as Total Focusing Method (TFM) (Holmes, Drinkwater, \& Wilcox, 2005).

The dark side of the simplicity of the algorithms being used is that the quality of flaws images is not always sufficiently high. They oftenly have many glares formed by re-scattered pulses and by pulses appeared in result of wave type transformation during the re-scattering on heterogeneities. Besides this, it is not always possible to make definite conclusion on the deflector's form, as it is the restored image of only the part of the deflector's edge which actually had reflected the pulses received in acquisition area. Therefore, there must, with not claim of completeness, be mentioned the existing methods of getting scatterers images based on a more precise solution of the inverse problem of scattering. Here are some of them: the MUltiple SIgnal Classification (MUSIC) (Devaney, 2000), the iterative algorithm for solving the inverse boundary problem of ultrasound scattering on the cavity in an isotropic solid body (Buro \& Prudnikova, 1999), Novikov-Henkin algorithm (Novikov, \& Henkin,1987; Novikov, 1986; Burov, Vecherin, Morozov, \& Rumyantsev, 2010), methods based on the acquisition of echo signals appearing due to nonlinear effects of second and third order (Burov, Gurinovitch, Rudenko, \& Tagunov, 1994; Burov \& Shmelev, 2009). It is worth mentioning a very promising direction for solving inverse problems, which becomes in more and more demand as far as the computing power grows. The solution of the inverse coefficient problem for the scalar wave equation (Goncharsky \& Romanov, 2011) is based on the possibility of the direct calculation of the gradient of the function closure via the solution of the so-called conjugate problem for partial derivatives equation by using the finite difference method. By turning to the vector version of the wave equation, it seems possible that all effects of propagation and scattering of sound would be taken into account. The appearance of run-round impulse, multiple re-scattering and wave type transformation of waves types will give information, which, at unilateral access, will allow to restore the field of density and elastic coefficients values in the inspected object, by which it will be possible to determine not only the entire boundary of the deflector, but also the properties of its material. The algorithms mentioned are quite complex in practical implementation, but, with the increasing of computing power, they will be actively used in the systems of mass ultrasound expert testing.

There is a number of mathematical methods not associated with solving acoustic inverse problem but enabling to improve the quality of deflectors images. Thus, to produce images of super resolution (exceeding the Rayleigh limit), the mathematical algorithms based on the extrapolation of temporal and spatial spectra of signals are used. For example, there is the iterative Gershberg-Papoulis algorithm (Gershberg, 1974; Lasaygues \& Lefebvre, 1998; Papoulis, \& Chamras, 1979; Bazulin, 1991; Pickalov, \& Kazantsev, 2008), or algorithm of signal spectrum extrapolation of echo based on building its AR-model (Marple, 1987; Faur, Morisseau, \& Poradis, 1998; Box, Jenkins, \& Reinsel, 1994); Bazulin, 1993). Gershberh-Papoulis algorithm is used for both echo signals spectra extrapolating, which leads to an increase in the longitudinal image resolution, and for extrapolating the complex spectrum of the image, which leads to the both longitudinal and transverse resolution enhance. As the use of the Gershberg-Papoulis (Papoulis, \& Chamras, 1979) method assumes the use of image-cutting-off operation at about $30 \%$ of the maximum value, this leads to a loss of information on the small amplitude scatterers. Extrapolation of echo signal spectrum in course of its the constructing of its AR-model leads to an increasing mainly the longitudinal, but not transverse resolution of the restored image. In article (Wan, Balasundar, \& Mandayam, 2003) a method for processing the echo signals using wavelet technology to achieve super-resolution is offered. The 
mentioned methods belong to the field of signal and image processing without taking into account specific features of nondestructive ultrasonic testing.

The maximum entropy method (MEM) is an outstanding one among the methods of solving inverse problems. In article (Freiden, 1972) the possibility is shown of achieving super-resolution in the images obtaining system using entropy as a stabilizing factor in the Tikhonov regularization method context (Tikhonov \& Arsenin, 1986). Investigations have confirmed the effectiveness of MEM practical application in restoring images tomography, radio astronomy(Wernecke, \& D`Addario, 1977; Baykova, 2008), nuclear magnetic resonance (NMR) (Hore, 1991), as well as in ultrasonic testing (Battle, 1999; Bazulin, \& Bazulin, 2005). In article (Bazulin, 2010) MEM is used for solving the inverse scattering problem in single-dimensional option selected in order to account for re-scattering pulses at pointlike discontinuities.

This article explores the possibility of using MEM in ultrasonic nondestructive testing for image reconstructing from the measured set of deflectors echo signals, considering their deflecting from uneven boundaries of the inspected object. In this paper, the second section is proposed to use MEM for reconstruction the image reflectors during of ultrasonic testing. In the third section describes the method of calculation of the ultrasonic field from a pointlike scatterer with account of multiple reflected pulse from the boundaries of the inspected object. The fourth section presents the results of the application of MEM to reconstruct the image reflectors and for compression of complex signals.

\section{Maximum Entropy Method (MEM)}

The solving the inverse scattering problem means finding the $\varepsilon(\mathbf{r})$ function describing the deflective properties of inhomogeneity within the $S_{t}$ area, by using both the known $q\left(\mathbf{r}_{t}, t\right)$ field sources located within the $S_{t}$ area and the scattered field $p\left(\mathbf{r}_{r}, t\right)$ measured over the $S_{r}$ area. This reverse problem is nonlinear one (Gorjunov \& Saskovets, 1989), since, besides the unknown parameters of $\varepsilon(\mathbf{r})$ inhomogeneity, the unknown total field $p(\mathbf{r}, t)$ in the entire region of interest (ROI) has to be determined. In the practice of ultrasonic testing the Born approximation is commonly used where the scattered field amplitude is assumed much smaller than that one of the incident field, i.e. $p(\mathbf{r}, t) \ll p_{i}(\mathbf{r}, t)$, which, from a strictly mathematical point of view, is oftenly not true.

Let the solution of the direct problem, i.e. the calculating the scattered field $p\left(\mathbf{r}_{r}, t\right)$ by the known $q\left(\mathbf{r}_{t}, t\right)$ and $\varepsilon(\mathbf{r})$ functions be formally written down as follows

$$
p\left(\mathbf{r}_{r}, t\right)=P\left(\varepsilon(\mathbf{r}), q\left(\mathbf{r}_{t}, t\right)\right)
$$

$\varepsilon(\mathbf{r})$ - the function describing the deflective properties, $q\left(\mathbf{r}_{t}, t\right)$ - the known field sources, $p\left(\mathbf{r}_{r}, t\right)-$ the scattered field. As the direct problem is linear or, as in the case of the Born approximation, can be linearized, the equation (1) can be written in the following matrix form:

$$
p=G \varepsilon+n,
$$

where matrix $G$ describes the propagation of ultrasonic waves from their sources belonging to $S_{t}$ area to the pointlike deflector and then to the $S_{r}$ acquisition area, the vector $n$ being the noise of measurements. Since $G$ matrix is usually poor-conditioned, there are, besides simple matrix inversion, some other solution options. One of them is finding an $\hat{\varepsilon}$ estimate as the solution of unconstrained optimization, a criterion of the quality of the reconstructed image being the square of discrepancy solution

$$
\chi^{2}(\hat{\varepsilon})=\|G \hat{\varepsilon}-p\|=(G \hat{\varepsilon}-p)^{T}(G \hat{\varepsilon}-p)
$$

where symbol $T$ denotes the transpose operation. Estimate $\hat{\varepsilon}$ can be written as

$$
\hat{\varepsilon}=\underset{\hat{\varepsilon} \in R^{N_{i}, x_{i, z}}}{\arg \min }\left(\chi^{2}(\hat{\varepsilon})\right) .
$$

The solution of the inverse problem in the form of (4) is called the method of least squares (Helstrom, 1967). To solve the problem of finding out the (4) minimum with the maximum speed, it seems better to use the second-order methods (Letova \& Panteleev, 1998). Their application requires a knowledge of the gradient and Hessian of the formula (3), which is calculated by the following formula

$$
\begin{gathered}
\nabla \chi^{2}(\varepsilon)=2 G^{T}(G \varepsilon-p) \\
\nabla \nabla \chi^{2}(\varepsilon)=2 G^{T} G .
\end{gathered}
$$


In terms of article(Tikhonov \& Arsenin, 1986), the solving the degenerated system of linear algebraic equations (4) with respect to $\varepsilon$, provides a minimum $\chi^{2}$ discrepancy and is called pseudosolution. There may be the infinite number of pseudosolutions, and their parameters such as resolution, speckle noise level and etc. in general case may appear to be far from ideal. In article (Lingvall, Olofsson, Wennerström, \& Stepinski, 2004) the LSM method was applied to restore the images of the scatterers by their echo signals in the numerical experiment, and that was called Extended Synthetic Aperture Focusing Technique (ESAFT).

The clear regularized solution of equation (4) does exist, the one valid for the case of non-square matrixes being

$$
\hat{\varepsilon}=\frac{G^{T}}{G G^{T}+\gamma E} p,
$$

where $E$ is the diagonal matrix, $\gamma$ is spectral density of the noise energy. This formula is structurally equivalent to the formula describing Wiener filter (Vasilenko, 1986) in the frequency domain. Using (7) allows to obtain high-quality images, but in cases of low level of noise. In matrix form the evaluation of function $\hat{\varepsilon}$ can be represented analogously with a correlation formula

$$
\hat{\varepsilon}_{c}=G^{T} p .
$$

The inverse problem is considered incorrect in the Hadamard sense (Tikhonov \& Arsenin, 1986), unless all of the three conditions are not fulfilled: for any element $p \in \mathrm{P}$ there is a solution $\varepsilon \in \mathrm{E}$, the solution is determined unequivocally and the problem is stable within the spaces (E,P). Unfortunately, the problem (2) is incorrect (Devaney, 2000). To solve alike problems, Tikhonov (Tikhonov \& Arsenin, 1986) has developed a method of regularization which justifies the replacement of the problem in the form (2) by the optimization problem stable to small changes in input data $p$

$$
\hat{\varepsilon}_{\alpha}=\underset{\hat{\varepsilon} \in R^{N, x^{N} x_{i, z}}}{\arg \min }\left(\chi^{2}(\hat{\varepsilon})+\alpha \Omega(\hat{\varepsilon})\right)
$$

where $\chi^{2}(\varepsilon)=\left\|P\left(\hat{\varepsilon}(\mathbf{r}), q\left(\mathbf{r}_{t}, t\right)\right)-p\left(\mathbf{r}_{r}, t\right)\right\|$ is the square of solution discrepancies in the metric defined by the specifics of the problem, $\Omega(\varepsilon)$ is a stabilizing function. The sense of using the stabilizing functions lays in possibility to consider any a priori information about the solution and thereby to narrow the area of searching for solutions.

The entropy of $\hat{\varepsilon}$ function can be used as the stabilizing function $\Omega(\hat{\varepsilon})$. Initially statistical physics concept of entropy, with symbol $S$ especially assigned for it, was introduced in 1865 by R. Clausius to denote a function describing the state of a thermodynamic system. In 1948, Shannon (Shannon, 1984) used the notion of entropy to assess the information volume of messages consisting of a finite set of characters of a certain alphabet. The text message of finite length contains the maximum amount of information, provided any sign of the message is followed by any alphabet character in arbitrary way and with equal probability. From a combinatorial point of view, such a message allows to create the maximum number of different character combinations with a limited set of alphabetic symbols. From the point of view of increasing the images resolution, using the entropy function as regularizing one allows to transfer the search for solutions on a set of images, in which the count with any value may be with any count, that is, the limitation on the solution front steepness is removed. Thus, restoring the image by linear method using the formula (4), the resolution remains practically unchanged, i.e. letter «Z» will never be followed by letter «X», but after regularization the front steepness can rise - this means that next to the letter «X letter of the alphabet. In case the entropy is applied as a stabilizing function, formula (9) can be rewritten as

$$
\hat{\varepsilon}_{\alpha}=\underset{\hat{\varepsilon} \in R^{N_{i, x} \cdot N_{i, .}}}{\arg \min }\left(\chi^{2}(\hat{\varepsilon})-\alpha H(\hat{\varepsilon})\right),
$$

where $H(\hat{\varepsilon})$ is the entropy of a set of discrete independent random variables. This is defined for the case of real, non-negative $\hat{\varepsilon}_{i}$ as

$$
H(\hat{\varepsilon})=-\sum_{i=1}^{N_{i, X_{i, z}}^{N_{i, z}}} \hat{\varepsilon}_{\dot{i}} \ln \hat{\varepsilon}_{\dot{i}}=-\Omega(\hat{\varepsilon}),
$$

where $N_{i, x} N_{i, z}$ is the number of points in the reconstructed image along the axis $x$ and axis $z$. In practice, the so-called cross-entropy (Kullback, 19668) is oftenly used 


$$
H(\hat{\varepsilon})=-\sum_{i=1}^{N_{i} N_{i z}} \hat{\varepsilon}_{i} \ln \frac{\hat{\varepsilon}_{i}}{m_{i}},
$$

where $m$ is either an priori model or an estimation of the form of solution $\varepsilon$. In the simplest model a constant $e \mu$ can be used, where $\mu$ is understood as the estimated mean intensity of the image background. In this article entropy of variable sign $\hat{\varepsilon}$ function was calculated by the formula (Maisinger, Hobson, \& Lasenby, 2004)

$$
\begin{gathered}
z_{i}=\sqrt{\hat{\varepsilon}_{i}^{2}-4 \mu^{2}}, \\
H_{ \pm}(\hat{\varepsilon})=-\sum_{i=1}^{N_{i, x} N_{i, z}}\left(z_{i}-2 \mu-\varepsilon_{i} \ln \frac{z_{i}+\varepsilon_{i}}{2 \mu}\right) .
\end{gathered}
$$

For efficient searching for the minimum of multivariable function by the second-order methods, the gradient and Hessian expressions (12) are to be calculated in the form

$$
\frac{\partial H_{ \pm}(\hat{\varepsilon})}{\partial \hat{\varepsilon}_{i}}=-\ln \left(\frac{z_{i}+\hat{\varepsilon}_{i}}{2 \mu}\right), \quad \frac{\partial^{2} H_{ \pm}(\hat{\varepsilon})}{\partial\left(\hat{\varepsilon}_{i}\right)^{2}}=-\frac{1}{z_{i}} .
$$

It is clear from equation (13) that Hessian represents a diagonal matrix. This obstacle can speed up optimization algorithms execution and, besides this, lower RAM volume requirements.

The main methodological problem of MEM practical application is the selection of the following algorithm parameters: evaluation of the background amplitude $\mu$ and the regularization parameter $\alpha$. There are both exact and empirical methods for selecting the regularization parameter: residual method (Tikhonov \& Arsenin, 1986; Morozov, 2003), the method of cross validation (Van Kempen \& Van Vliet, 2000), the problem of generalized minimization (Morozov, 2004), L-curve method (Hansen, 2001), adaptive method (Valyashko \& Strakhov, 1981) and others. However, some methods are iterative and, therefore, require significant computing resources, while others need additional information, for example, the value of the noise variance $\sigma(n)$ which cannot always be measured with sufficient accuracy. And the authors of the article (Francini, Höft, \& Santosa, 2006) after having carried out a set of numerical experiments just compiled a table that relates $\alpha$ to the magnitude of the noise variance $\sigma(n)$. An algorithm called adaptive estimation was used in the article (Bazulin, \& Bazulin, 2005). However, in many practical works on the application of MEM, the problem (9) is solved for a set of $\alpha$ Lagrange coefficients, then the best solution is selected from a set of estimates $\hat{\varepsilon}_{\alpha}$ according to a predetermined criterion. In this article the image having maximum focusing was chosen from a set of estimates $\hat{\varepsilon}_{\alpha}$ for a set $\left\{\alpha_{1}, \alpha_{2}, \ldots \alpha_{N_{\alpha}}\right\}$.

\section{Calculation of Matrix G Considering Multiple Reflections From the Boundaries of the Inspected Object}

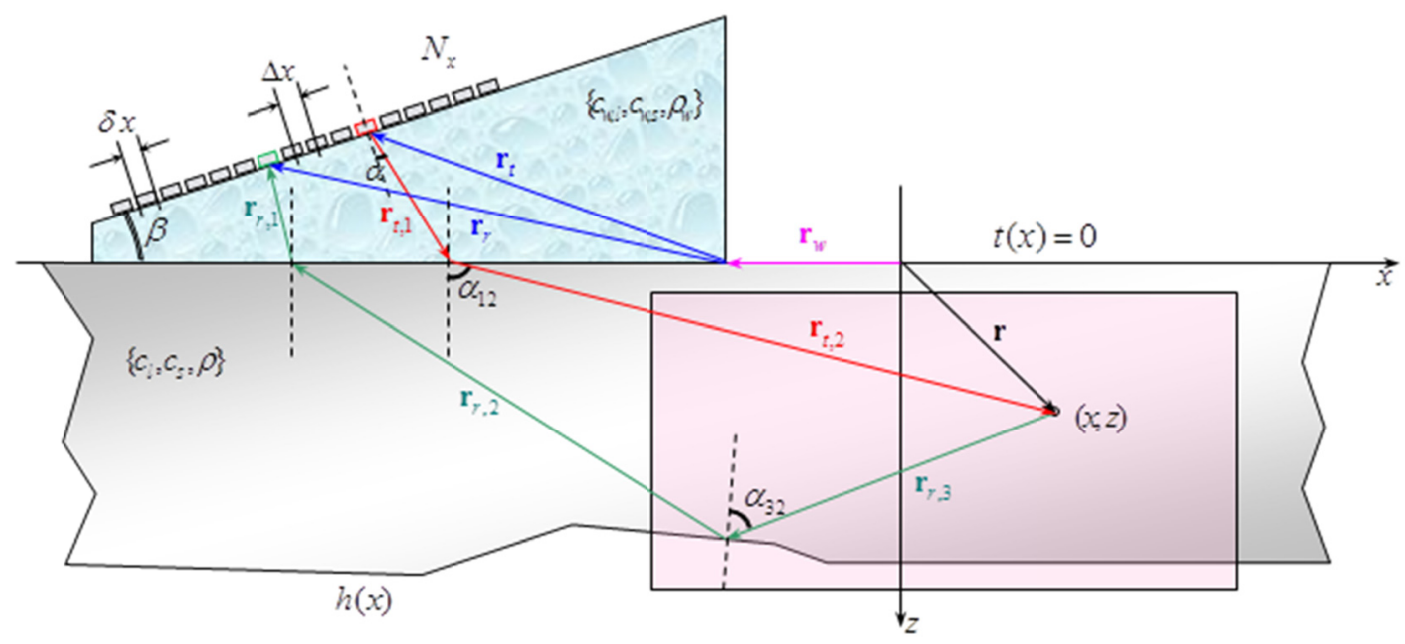

Figure 1. Illustration for the calculating the $G\left(\mathbf{r}_{t}, \mathbf{r}, t\right)$ function for the emitter and the $G\left(\mathbf{r}_{r}, \mathbf{r}, t\right)$ function for the receiver 
Suppose that for the emitting and receiving the ultrasonic waves an antenna array mounted on a prism is used which has a $\beta$ tilt angle and is made of the material featuring velocities of longitudinal and transverse waves and a density of $\left\{c_{w, l}, c_{w, s}, \rho_{w}\right\}$ (Figure 1). The antenna array consists of $N_{t}=N_{r}$ elements of $\delta x$ size located at a $\Delta x$ distance from each other. Let us designate sound velocity and density of the inspected object as $\left\{c_{l}, c_{s}, \rho_{w}\right\}$. The location of transmitters and receivers relative to the center of the prism front edge are described by $\mathbf{r}_{t}$ and $\mathbf{r}_{r}$ vectors shown in Figure 1 with blue arrows. The prism front edge is shifted from the center of the $x z$ coordinate system by the value of $\mathbf{r}_{w}=\left(x_{w}, 0\right)$. The antenna array with a single element $N_{t}=N_{r}=1$ can be regarded as a single element transducer.

To determine the elements of matrix $G$ in the formula (2), it is necessary to calculate the field in the $S_{r}$ area of acquisition for the case when the pointlike deflector is placed at any arbitrary point of ROI and the transmitter is put in $S_{t}$ area, ROI being shown with pink rectangle in Figure 1.

The trajectory of pulse movement in case of emission (shown in Figure 1 with red arrows) can be described as a sequence of vectors $\left\{\mathbf{r}_{t}\right\}=\left\{\mathbf{r}_{t, 1}, \mathbf{r}_{t, 2}, \ldots \mathbf{r}_{t, m_{t}+1}\right\}$, where $m_{t}$ is the number of deflections from the boundaries of the specimen, in case of acquisition - as a sequence $\left\{\mathbf{r}_{r}\right\}=\left\{\mathbf{r}_{r, 1}, \mathbf{r}_{r, 2}, \ldots \mathbf{r}_{r, m_{r}+1}\right\}$, where $m_{r}$ is the number of deflections from the boundaries of the specimen in course of acquisition (in Figure 1 the trajectory is shown with green arrows). The first vector of such a sequence always corresponds to the path of sound propagation in the prism. In case of emission the velocity of pulse propagation long the path is defined by the list $\left\{c_{t}\right\}=\left\{c_{t, 1}, c_{t, 2}, \ldots c_{t, m_{t}+1}\right\}$, and in case of acquisition - by list $\left\{c_{r}\right\}=\left\{c_{r, 1}, c_{r, 2}, \ldots c_{r, r_{r}+1}\right\}$. The first element in these lists is in all cases $c_{w, l}-$ the longitudinal wave velocity in the prism. The remaining elements can take a value of either longitudinal $c_{l}$ or transverse $c_{s}$ velocity of sound in the inspected object, thus allowing taking into account the effect of the transformation of the wave type once it is reflected from the boundary. The minimally possible rates list for the case $m_{t}=0$ or $m_{r}=0$ looks like $\left\{c_{t}\right\}=\left\{c_{w, l}, c_{s}\right\}$ or $\left\{c_{r}\right\}=\left\{c_{w, l}, c_{s}\right\}$ and corresponds to the option of working with a transverse wave in a direct beam.

For describing the acoustic schemes in which the reflection of ultrasonic pulses from both the bottom and surface of the inspected object take place, we will use the following designations: such events as surface refraction and surface deflection will be designated by letter $\mathrm{T}$, and the deflection from the bottom will be denoted by letter $\mathrm{B}$. Type of waves after the event is occurred will be denoted by the letters L (longitudinal) and S (shearing). Expression $\mathrm{T}(\mathrm{S})-\mathrm{T}(\mathrm{L})$ denotes a common direct beam on a transverse wave in case of emission and the direct beam but on the longitudinal wave $\left(m_{t}=0, m_{r}=0\right)$ in case of acquisition. Acoustic scheme TB(LL)-TBT(SSS) describes a situation where, in case of emission, only the radiation beams once reflected on the longitudinal wave are considered, and, in case of acquisition - only the ones doubly reflected on the transverse wave $\left(m_{t}=1, m_{r}=2\right)$; acoustic scheme T(L)-TB(SL) corresponds to that one called «self tandem» (Ermolov \& Lange, 2004) $\left(m_{t}=0\right.$, $\left.m_{r}=1\right)$. The beam shown with the solid line corresponds to transverse wave propagation, and the dashed line longitudinal wave propagation. Figure 1 represents a scheme of the rays propagation in correspondence with acoustic scheme $\mathrm{T}(*)-\mathrm{TB}(* *)$

To build either a function $G\left(\mathbf{r}_{t}, \mathbf{r}, t\right)$ for $m_{t}$ reflections from the inspected object boundary at emission or function $G\left(\mathbf{r}_{r}, \mathbf{r}, t\right)$ for $m_{t}$ deflections at the acquisition, one may use the geometrical optics approximation (Kravtsov \& Orlov, 1980; Gengembre, 2003). Since the sizes of the antenna elements are comparable to the wave length, they may be considered to be the pointlike emitters - receivers with the $\Theta(\alpha)$ directivity diagram in prism, where $\alpha_{t}, \alpha_{r}$ is beam angle to the normal of piezoelectric element of the emitter or receiver. Having taken into account these approximations, the functions $G\left(\mathbf{r}_{t}, \mathbf{r}, t\right)$ and $G\left(\mathbf{r}_{r}, \mathbf{r}, t\right)$ can be written as

$$
\left\{\begin{array}{l}
G\left(\mathbf{r}_{t}, \mathbf{r}, t\right)=A\left(\mathbf{r}_{t}, \mathbf{r}\right) s\left(t-t_{t}\left(\mathbf{r}_{t}, \mathbf{r} ; m_{t}\right)\right), t_{t}\left(\mathbf{r}_{t}, \mathbf{r} ; m_{t}\right)=\sum_{n=1}^{m_{t}+1} \frac{\mathbf{r}_{t, n} \mid}{c_{n}}, \\
A\left(\mathbf{r}_{t}, \mathbf{r}\right)=\frac{\Theta\left(\alpha_{t}\right) D_{w, o}\left(\alpha_{12} ; c_{w, l}, c_{t, 1}\right) \prod_{n=2}^{m_{t}} V\left(\alpha_{n} ; c_{t, n}, c_{t, n+1}\right)}{R\left(\left\{\mathbf{r}_{t}\right\},\left\{c_{t}\right\}, h(x)\right)} \\
G\left(\mathbf{r}_{r}, \mathbf{r}, t\right)=A\left(\mathbf{r}_{r}, \mathbf{r}\right) s\left(t-t_{r}\left(\mathbf{r}_{r}, \mathbf{r} ; m_{r}\right)\right), t_{r}\left(\mathbf{r}_{r}, \mathbf{r} ; m_{r}\right)=\sum_{n=1}^{m_{r}+1} \frac{\left|\mathbf{r}_{r, n}\right|}{c_{n}}, \\
A\left(\mathbf{r}_{r}, \mathbf{r}\right)=\frac{\Theta\left(\alpha_{r}\right) D_{o, w}\left(\alpha_{12} ; c_{w, l}, c_{r, 1}\right) \prod_{n=2}^{m_{r}} V\left(\alpha_{n} ; c_{r, n}, c_{r, n+1}\right)}{R\left(\left\{\mathbf{r}_{r}\right\},\left\{c_{r}\right\}, h(x)\right)},
\end{array}\right.
$$


where $s(t)$ is the radiated impulse, $t_{t}\left(\mathbf{r}_{t}, \mathbf{r} ; m_{t}\right)$ and $t_{r}\left(\mathbf{r}_{r}, \mathbf{r} ; m_{r}\right)$ - times of impulse travelling along the path of the radiation $\left\{\mathbf{r}_{t}\right\}$ and reception $\left\{\mathbf{r}_{r}\right\}, D_{w, o}\left(\alpha_{12} ; c_{w, l}, c_{t, 1}\right)$ and $D_{o, w}\left(\alpha_{12} ; c_{w, l}, c_{r, 1}\right)$ - pressure transparence coefficients for the angle of refraction $\alpha_{12}$ during the wave propagating from the prism to the inspected object and back, $\left(c_{w, l}, c_{t, 1}\right)$ or $\left(c_{w, l}, c_{r, 1}\right)$ - a given pair of sound velocities (Figure 1) (Ermolov \& Basatskaya, 1989). Symbol $V\left(\alpha_{n} ; c_{t, n}, c_{t, n+1}\right)$ denotes the complex coefficient of deflecting from the inspected object edge, which is dependent of the type of incident and reflected wave $\left(c_{t, n}, c_{t, n+1}\right)$ for reflection with number $n$ (Brekhovskikh \& Godin, 1992). Changing of the pulse amplitude of the is described by the ray divergence function $R(\{\mathbf{r}\},\{c\}, h(x, y))$, the form of which is determined by the assumption that the flow of energy in a ray tube is constant. Thus, for calculation the elements of matrix $G$ in equation (2), we can use formula (14) and write the following equation

$$
G\left(\mathbf{r}_{t}, \mathbf{r}_{r}, \mathbf{r}, t\right)=B(\mathbf{r}) A\left(\mathbf{r}_{t}, \mathbf{r}\right) A\left(\mathbf{r}_{r}, \mathbf{r}\right) s\left(t-t_{t}\left(\mathbf{r}_{t}, \mathbf{r} ; m_{t}\right)-t_{r}\left(\mathbf{r}_{r}, \mathbf{r} ; m_{r}\right)\right),
$$

where $B(\mathbf{r})$ is the coefficient of deflection from the scatterer. And here a problem arises that points at the fundamental limitation of this very approach of deflector image restoration, since neither its shape nor its acoustic properties are known. Nevertheless, the following approximate solution to this problem seems to be proposed. Figure 2 is a schematic representation of the rays propagating from the source to the imaginary scatterer and then back to the receiver, the acoustic scheme being T(S)-TBT(SLL). The surface of the imaginary scatterer is shown with red line and the normal to it - with dotted black line. Real scatterer is shown with the broken black line. Having calculated the angle $\alpha_{\Delta}$ between the ray fallen on the point $\alpha_{\Delta}$ of the imaginary scatterer and the reflected ray, one can then find the angle of incidence $\alpha_{d}$ by formula

$$
\alpha_{d}=\alpha_{\Delta}-\arctan \frac{c_{r, m_{r}+1} \sin \alpha_{\Delta}}{c_{t, m_{t}+1}+c_{r, m_{r}+1} \cos \alpha_{\Delta}} .
$$

After the evaluation the of the angle of incidence $\alpha_{d}$ on the surface of an imaginary scatterer is made, it becomes necessary to get to know its acoustic properties in order to calculate the reflection coefficient $B(\mathbf{r})=V\left(\alpha_{d} ; c_{t, m_{+}+1}, c_{r, m_{r}+1}\right)$. Since its acoustic properties are unknown, the reflection coefficient will be calculated basing on the assumption that the scatterer has absolutely acoustically soft edge. But as for real cracks, there may occur an uncertain situation, as the crack may be actually filled with water or air, or scoria.

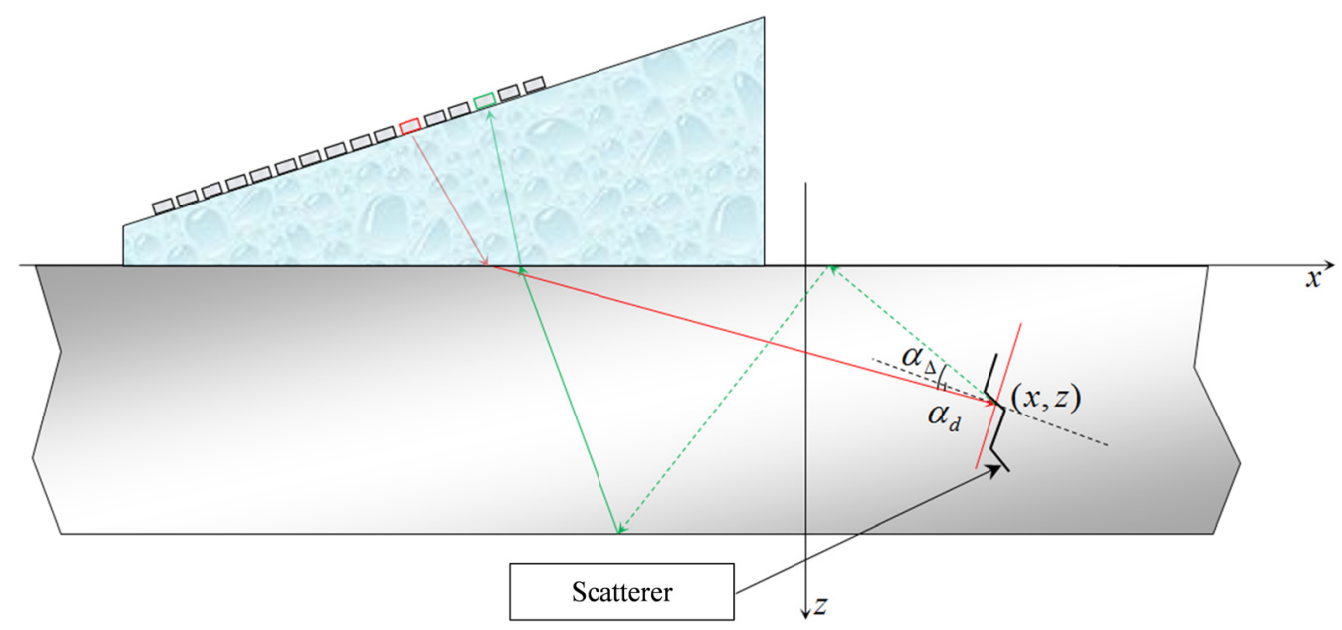

Figure 2. Determination of the angle of incidence for the acoustic scheme T(S)-TBT(SLL) on the imaginary scatterer (shown with red line) that does not coincide with the actual one (shown with a broken black line)

Formula (2) and (14) allow to calculate the field scattered by deflectors $\varepsilon(\mathbf{r})$ of known shape in the Kirchhoff approximation for a given sources function $q\left(\mathbf{r}_{t}, t\right)$. For this purpose it is necessary to add to the formula (2) and (14) the integration by vector $\mathbf{r}$ within the «illuminated» deflector surface. Equation (14) does not describe the effects of attenuation and dispersion, but these ones can be considered if necessary.

When making calculations using formula (14), there appears a certain problem of determining the ray paths in course of both emission $\left\{\mathbf{r}_{t}\right\}=\left\{\mathbf{r}_{t, 1}, \mathbf{r}_{t, 2}, \ldots \mathbf{r}_{t, m_{t}+1}\right\}$ and reception $\left\{\mathbf{r}_{t}\right\}=\left\{\mathbf{r}_{t, 1}, \mathbf{r}_{t, 2}, \ldots \mathbf{r}_{t, m_{t}+1}\right\}$. Some analytical solutions of this problem for the direct beam are known, but its analytical solution for an arbitrary number of reflections from uneven boundary of an object seems to be impossible. To determine the path of pulse propagation it is possible to use Fermat's principle (Born, \& Wolf, 1999), which states that the propagation of light or sound in a space of a given velocity distribution $c(x, z)$ from one point to another is a path along which light or sound passes within a 
minimum period of time. This variational approach makes it easy to take into account effects such as multiple reflections from uneven boundary of the inspected object and transformation of wave types in course of reflecting and refracting at the boundaries of different environments. However, it is usually implemented by means of an iterative procedure, which slows down the calculations rate.

To reduce the amount of echo signals to be processed, let us introduce the notion of switching matrix $\mathbf{C}$ of $N_{t} \times N_{r}$ dimensions. The expression $\mathbf{C}_{n m}=1$ means that it is the antenna array element numbering $n$ that is radiating, while the element numbering $m$ is receiving. For reducing the volume of echo can be measured only the upper (lower) triangle of matrix C (Samokrutov \& Shevaldykin, 2011). The characteristic form of the switching matrix $\mathbf{C}$ for 32-element antenna array at a random choice of 70 pairs $(m, n)$ is shown in Figure 3.

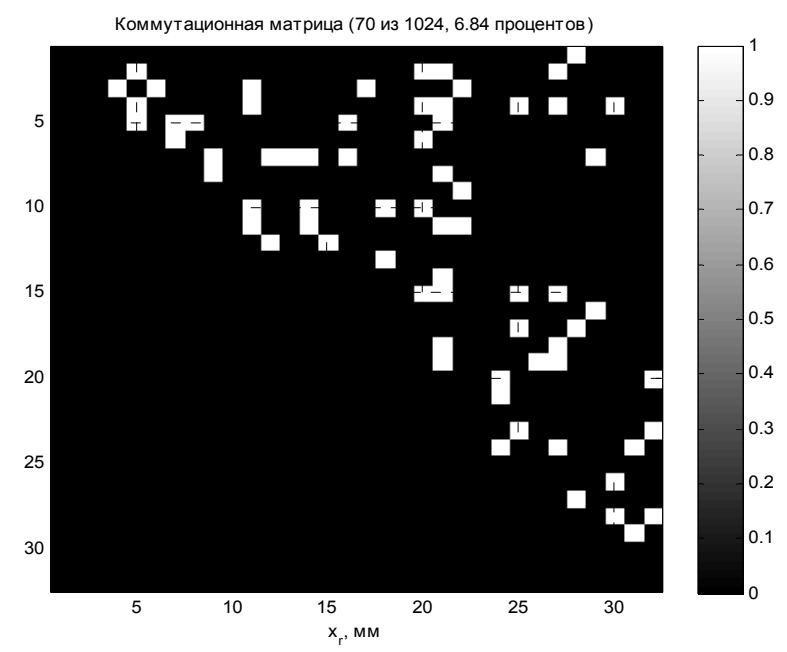

Figure 3. Typical view of the switching matrix $\mathbf{C}$

\section{Model experiments}

It should be noted that the more accurately echo signals $\hat{p}$ are calculated by a given estimate of the function $\hat{\varepsilon}_{\alpha}$, the better convergents the solution of the problem (9). Errors caused by inaccuracies of matrix $G$ calculation by formula (14) are called an operating noise. In this paper for calculating the matrix $G$ a simplified version of formula (14) was used in which the reference signal $s(t)$ of constant amplitude was shifted for the time $t_{t}\left(\mathbf{r}_{t}, \mathbf{r} ; m_{t}\right)+t_{r}\left(\mathbf{r}_{r}, \mathbf{r} ; m_{r}\right)$, and for acoustic schemes with an odd number of deflections T(S)-TB(SS) and TB(SS)-TBT( SSS) the reference signal was assumed to be equal to $-s(t)$ (Samokrutov \& Shevaldykin, 2011).

\subsection{Specimen With Six Inclined Saw Cuts (Frontal Resolution for Inclined Probe Testing)}

To register echo signals from the tops of six crack models each of $15 \mathrm{~mm}$ height, $0.05 \mathrm{~mm}$ opening and inclined at $60^{\circ}$ angle within a duralumin block, there was used a sloping single element probe operating on the longitudinal wave, with beam width of about 40 degrees and the central frequency of $2.5 \mathrm{MHz}$. The scan area is shown schematically in Figure 4 with several probe images. 


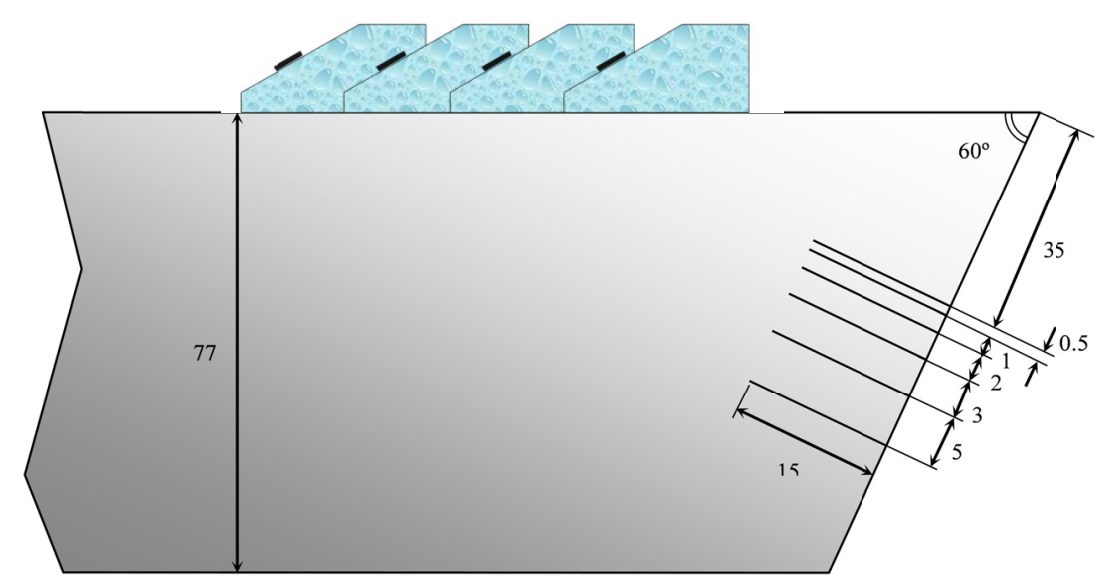

Figure 4. Specimen for testing of frontal resolution for inclined probe
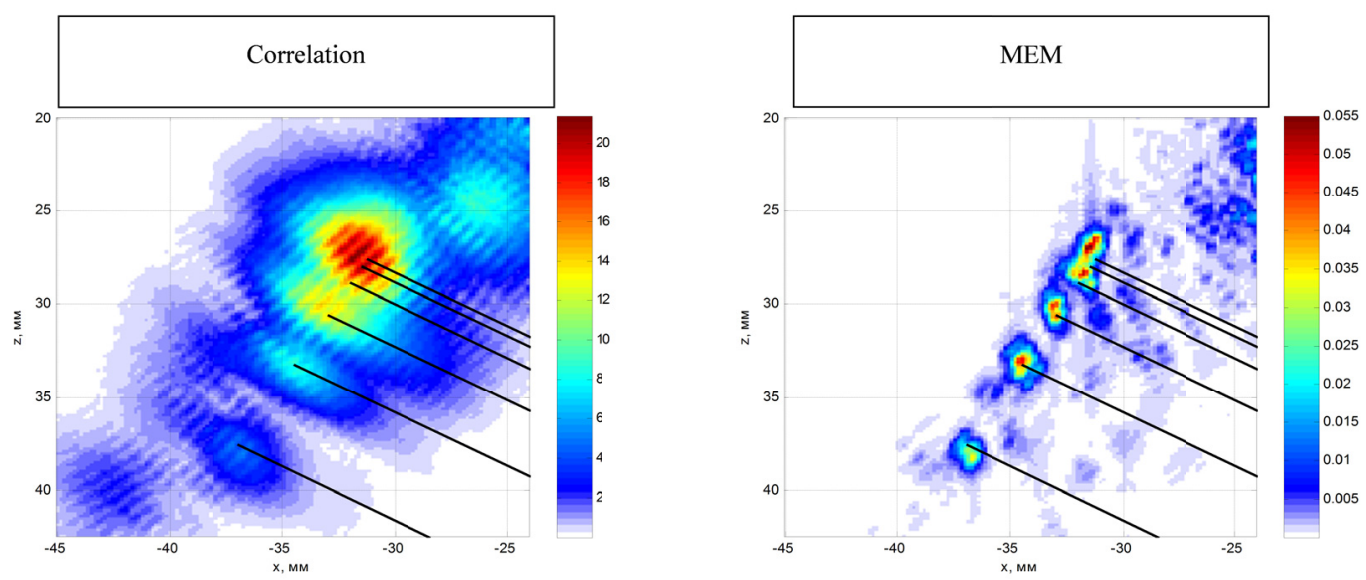

Figure 5. Images of the tops of six inclined saw cuts restored by correlation method and MEM

Figure 5 shows the images of the tops of six vertical saw cuts restored by correlation method using formula (8) (left picture), and the same ones restored by MEM using formula (2) and (9) in result of 20 iterations at $\alpha=0.05$ and $\mu=10^{-4}$. The images obtained by MEM method, if compared with the ones obtained by correlation method, has a nearly five time greater longitudinal resolution, nearly three times greater frontal resolution and signal/noise ratio increased by $14 \mathrm{~dB}$. Nevertheless, at a 0,7 level the image obtained by MEM failed to resolve tops of two cuts spaced $0.5 \mathrm{~mm}(0.2 \lambda)$ apart. Images in Figure 5 were obtained from 35 randomly selected echo signals, that is approximately $18 \%$ of the 190 all measured echo signals. Black lines on the figures designate a mask of six inclined sawcuts.

\subsection{Specimen of Pipeline of $800 \mathrm{~mm}$ Diameter With Nickel Plated Steel Welding}

A piece of a real pipeline of diameter of $800 \mathrm{~mm}$, thickness of $h=38 \mathrm{~mm}$ and with a cladding of about $5 \mathrm{~mm}$ thick was taken as a specimen (Figure 6). Then a nickel plated steel welding was made in the specimen. On one edge on the border «welding-base metal (perlite)» at the depths of 10, 20 and $30 \mathrm{~mm}$ three holes of $2.2 \mathrm{~mm}$ diameter (indicated by the numbers 1, 2 and 3 in (Figure 6) were made by side drilling method. 


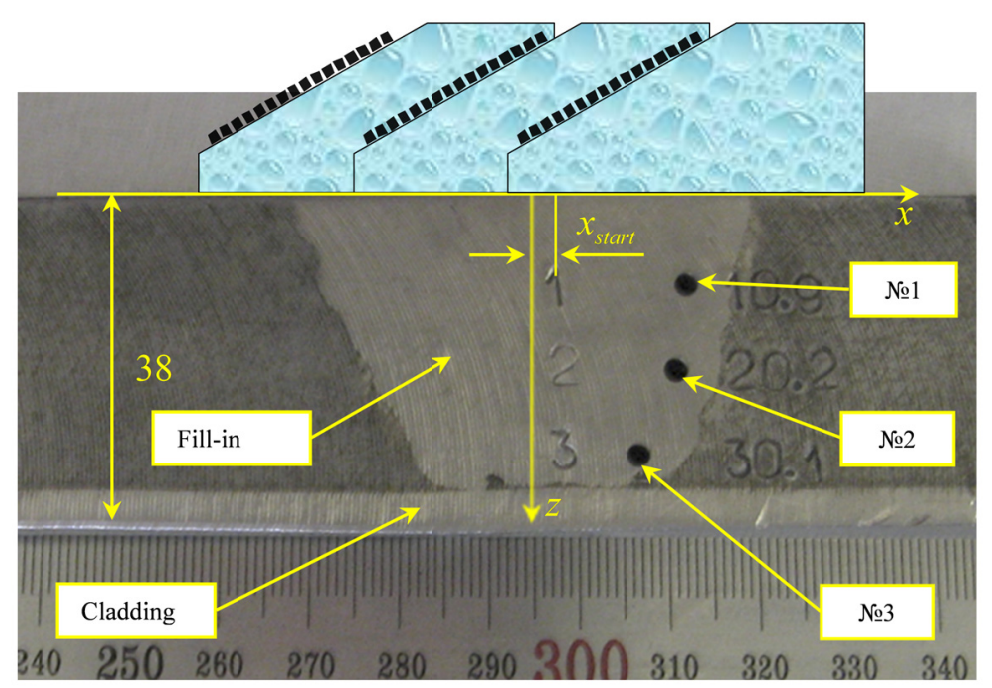

Figure 6 . The scheme of the pipeline specimen of $800 \mathrm{~mm}$ diameter, $38 \mathrm{~mm}$ thick, with nickel plated steel welding and three side drilled holes of $2.2 \mathrm{~mm}$ diameter

Because of strong ultrasonic wave attenuation in the nickel plated steel welding, echo signals were acquired on a longitudinal wave by a 20-element antenna array with $1.2 \mathrm{~mm}$ spacing and the operating frequency of $2.25 \mathrm{MHz}$ mounted on plexiglas prism of with the $20^{\circ}$ incline. Measurements were carried out in a triple scan mode when scanning in ten states with $1 \mathrm{~mm}$ pace from the point $x_{\text {start }}=2.9 \mathrm{~mm}$ along the front face of the prism. The scan area is shown schematically in Figure 6 with several images of antenna array on the prism. Because of the welding, in course of restoring the images of deflectors by TS-MC-SAFT (Brekhovskikh \& Godin, 1992) method and calculating the matrix $G$, it was only one acoustic scheme T(L)-T(L) that had been used. Figure 7 shows the images of the boundaries of side drilling holes and the bottom of the specimen restored by TS-MC-SAFT method. The left picture shows a partial image made for the first position of antenna array, and the right one - the image obtainer in result of coherent addition of the partial images of echo signals measured by a triple scan. It is clearly seen that after coherent summation of the partial image had been made, the signal/noise ratio increased by about 6 dB. All of $4000\left(10 \times 20^{2}\right)$ measured echo signals were used in restoring by TS-MC-SAFT method, the matrix $G$ having been formed by the principle outlined in section 4.3 . The images of the specimen bottom and the boundaries of side drilling holes are covered by the mask of black lines.
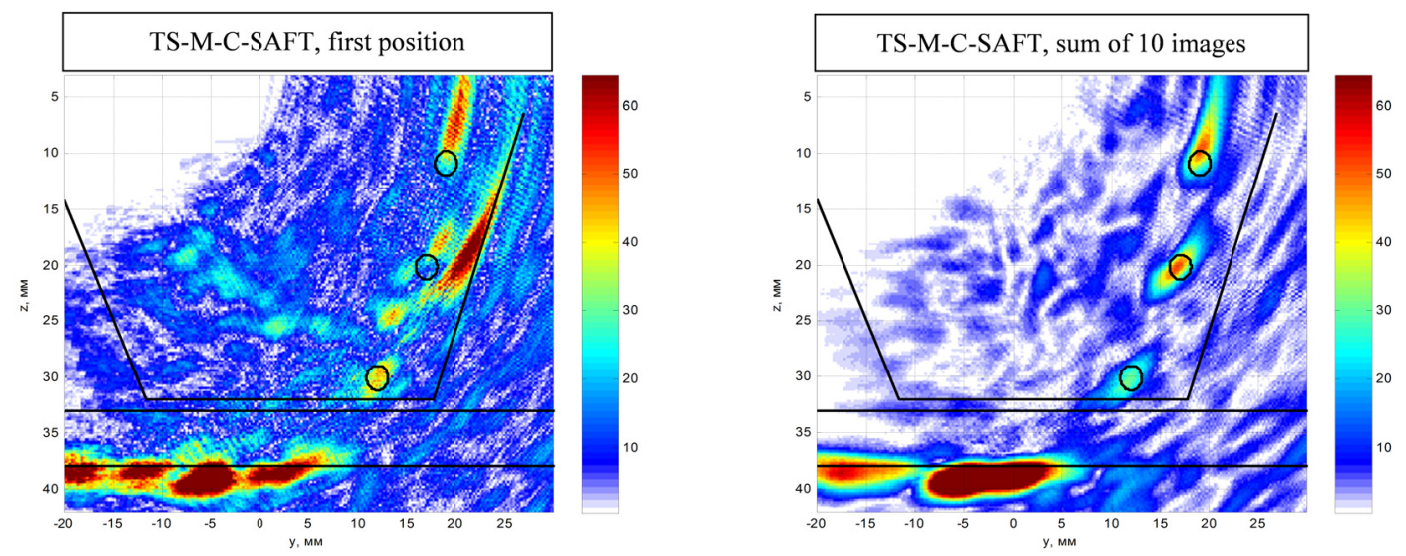

Figure 7. Images of the boundaries of side drilling holes and the bottom of the specimen restored by TS-M-C-SAFT method by echo signals measured in the triple-scanning mode

The left picture of Figure 8 shows images of the boundaries of side drilling holes and the bottom of the specimen restored by the correlation method using formula (8) for the random switching matrix $\mathbf{C}$ (Figure 3), and the 
right one - by MEM using formula (2) and (9), by the same echo signals after having made 10 iterations at $\alpha=15.0$ and $\mu=10^{-4}$. In comparison with the image obtained by the correlation method, frontal and longitudinal resolution of images obtained by MEM increased improved nearly twice, while the signal/noise ratio increased by more than $8 \mathrm{~dB}$. Images in Figure 8 were obtained from 300 randomly selected echo signals, that is $7.5 \%$ of all 4000 measured echo signals. That is, it takes 10 time less number of echo signals to effectively restore images by MEM, if compared with linear processing methods. Note that special methods were developed for working with incomplete data, one of them being a Compressive Sensing algorithm that uses $L_{1}$ -optimization methods for the so-called sparse signals (Granichin, 2009).

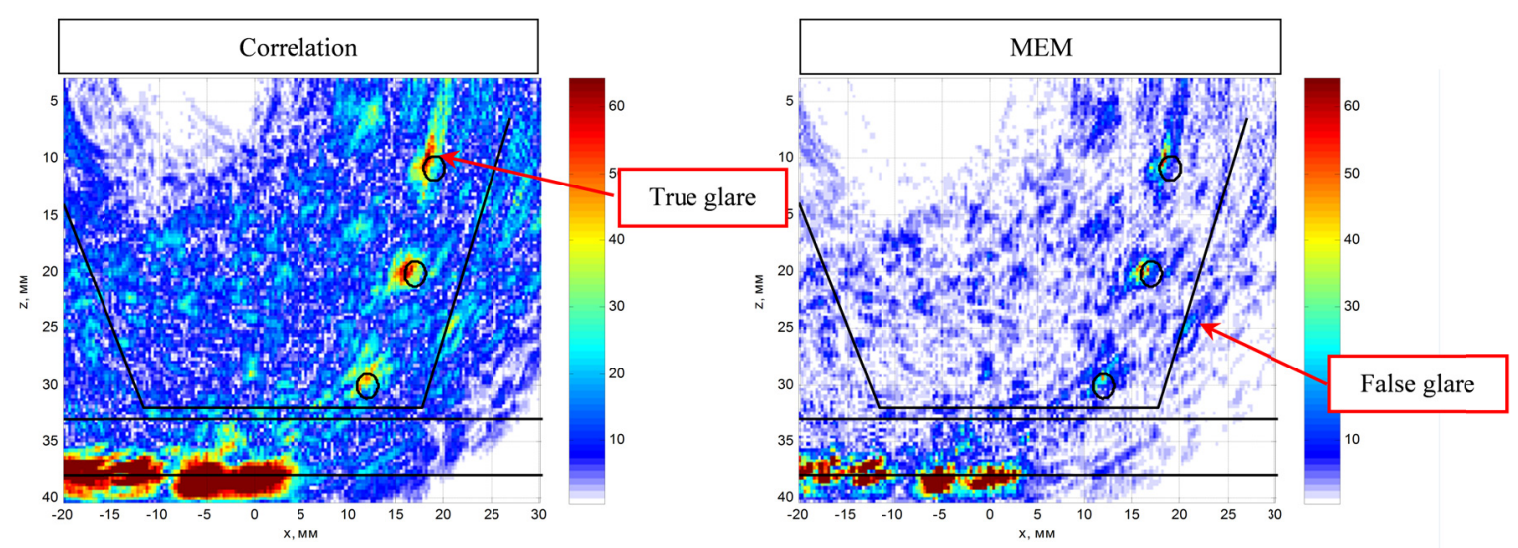

Figure 8. Images of the boundaries of side drilling holes and the bottom of the specimen restored by correlation method (left) and MEM (right)

Using of a random sampling of echo signals may lead to the appearing of false glares, which can be distinguished from the glares of deflectors in the way described below. It is necessary to get several random samples of echo signals for different options of the switching matrix $\mathbf{C}$ from the initial set of echo signals, and then to restore the image of deflector for each option. By comparing these images, it is possible to separate the false images from the glares corresponding to the real deflectors. The left of Figure 9 represents the coherent sum of images restored by the correlation method using formula (8), and the right - by MEM using formula (2) and (9), for seven options of the switching matrix $\mathbf{C}$. In comparison with the images shown on the left plate, the amplitude of the false images decreased by more than $6 \mathrm{~dB}$.
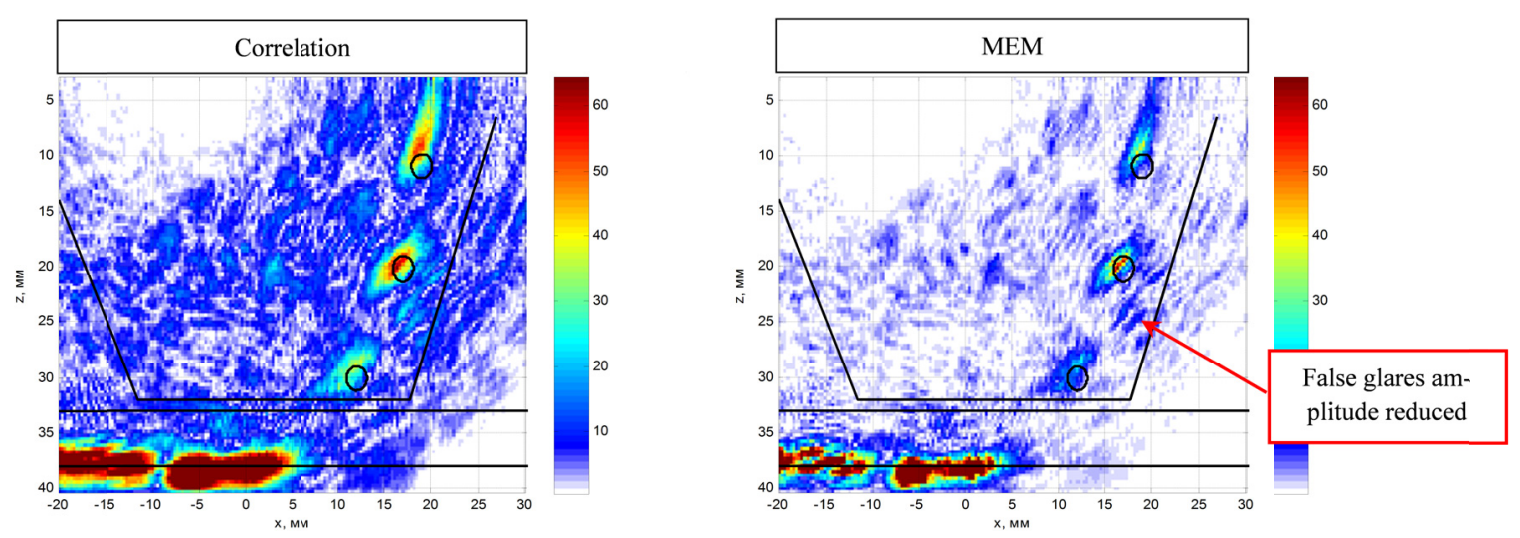

Figure 9. Images of the boundaries of side drilling holes and the bottom of the specimen restored by correlation method (left) and MEM (right), averaged over seven options of switching matrix $\mathbf{C}$ 


\subsection{The model of near-bottom crack with top at a depth of $12 \mathrm{~mm}$ in a specimen with an irregular bottom}

To demonstrate the feasibility of reconstructing the shape of deflectors when using the antenna array together with several acoustic schemes, the following simulation experiment was conducted.

Figure 10 shows a photo of the specimen of $h=18 \mathrm{~mm}$ thickness along with a model of bottom crack of a groove shape of $0.7 \mathrm{~mm}$ width with the top at $12 \mathrm{~mm}$ depth. To simulate a cone groove in the welded joint of pipeline, the steel specimen had an uneven bottom. For receiving the echo signals a 32-element antenna array was used with 1 $\mathrm{mm}$ step, working on operating frequency of $5 \mathrm{MHz}$ and mounted on rexolite prism of $35^{\circ}$ incline (Figure 10).

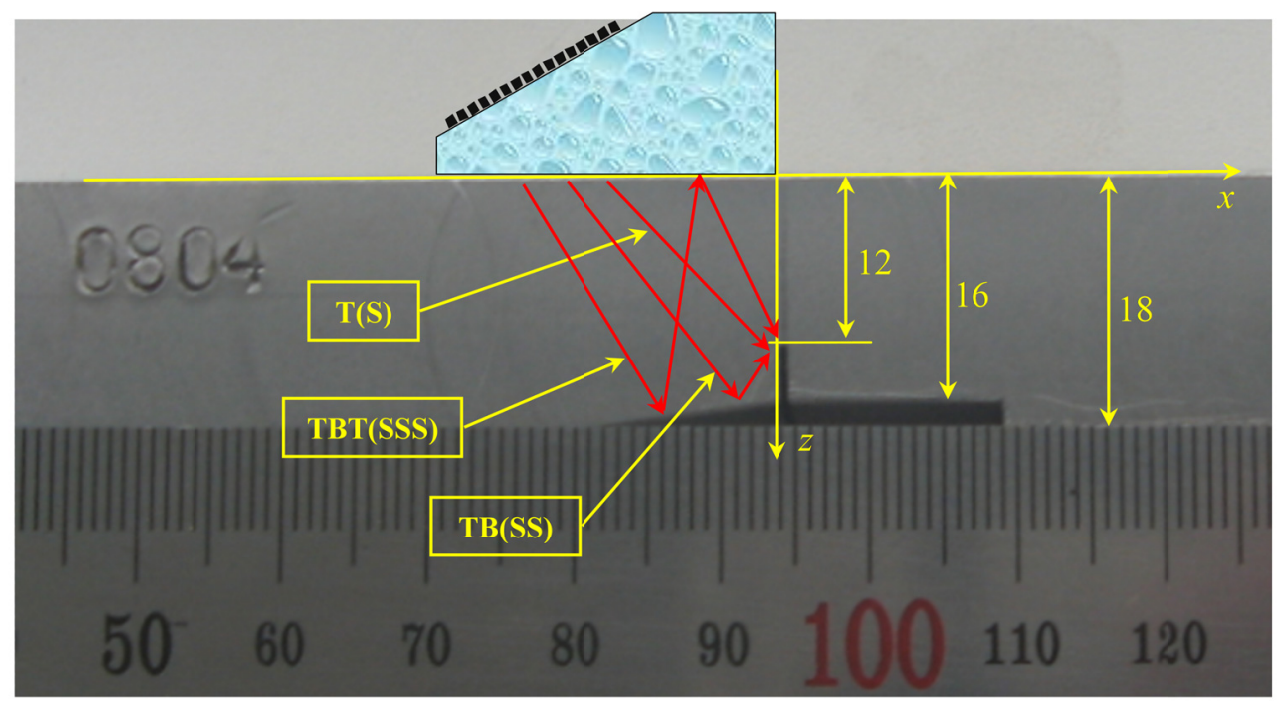

Figure 10. Photo of a specimen with a model of crack of a groove shape with $0.7 \mathrm{~mm}$ width with the vertex at a depth of $12 \mathrm{~mm}$. Red arrows schematically show the paths of three acoustic emission schemes: T(S), TB(SS) and TBT(SSS)

Figure 11 shows the images obtained by the TS-MC-SAFT method from the set of 1024 echo signals measured in dual scan mode. In the left panel of figure 11 the image obtained by one acoustic scheme $T(S)-T(S)$ is shown, and on the right panel - by the addition of the partial images according to acoustic schemes $T(S)-T(S)$, $\mathrm{T}(\mathrm{S})-\mathrm{TB}(\mathrm{SS})$ and $\mathrm{TB}(\mathrm{SS})-\mathrm{TB}(\mathrm{SS})$. The specimen bottom and saw cut contours are masked with the black lines. It can be stated that the vertically oriented crack is detected.
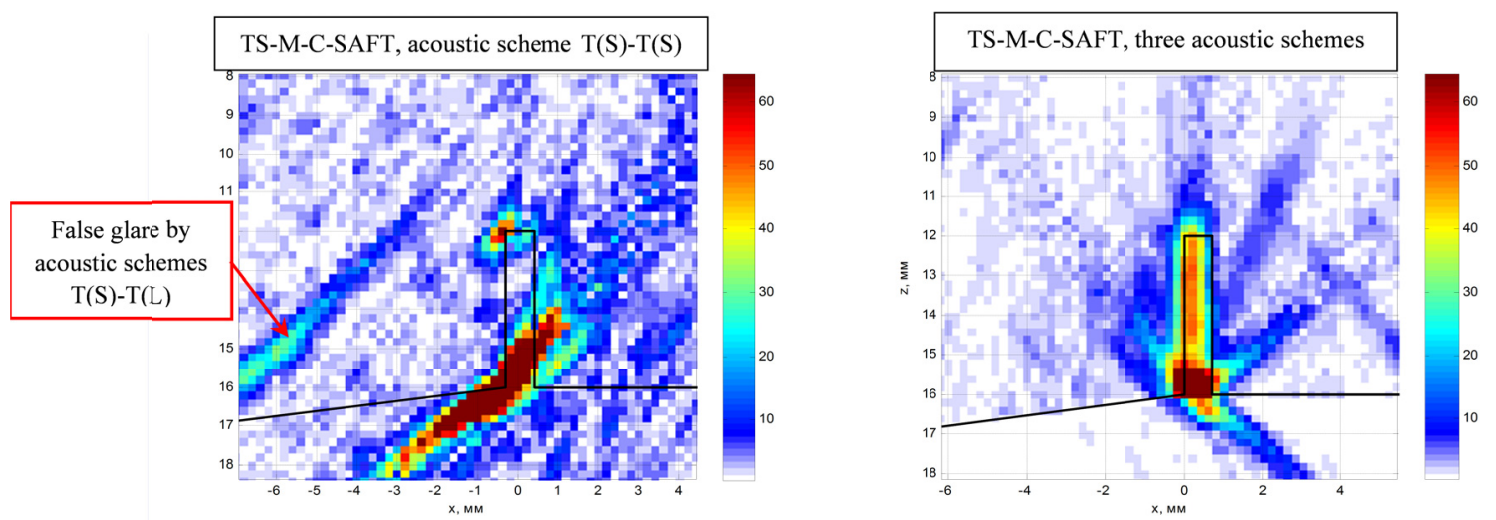

Figure 11. Boundary images of the model of a vertical crack restored by TS-MC-SAFT method by echo signals measured in dual scan mode 
Figure 12 shows an image of the boundary of the crack model restored by correlation method using formula (8) on the left, and on the right - MEM by formulae (2) and (9) after 10 iterations at $\alpha=1.0$ and $\mu=10^{-5}$ by acoustic scheme T(S)-T(S). The image of crack model obtained by MEM allows confidently resolve edges of the groove at a depth of $12 \mathrm{~mm}$, and the signal/noise ratio compared to the image obtained by the correlation method increased by $11 \mathrm{~dB}$.
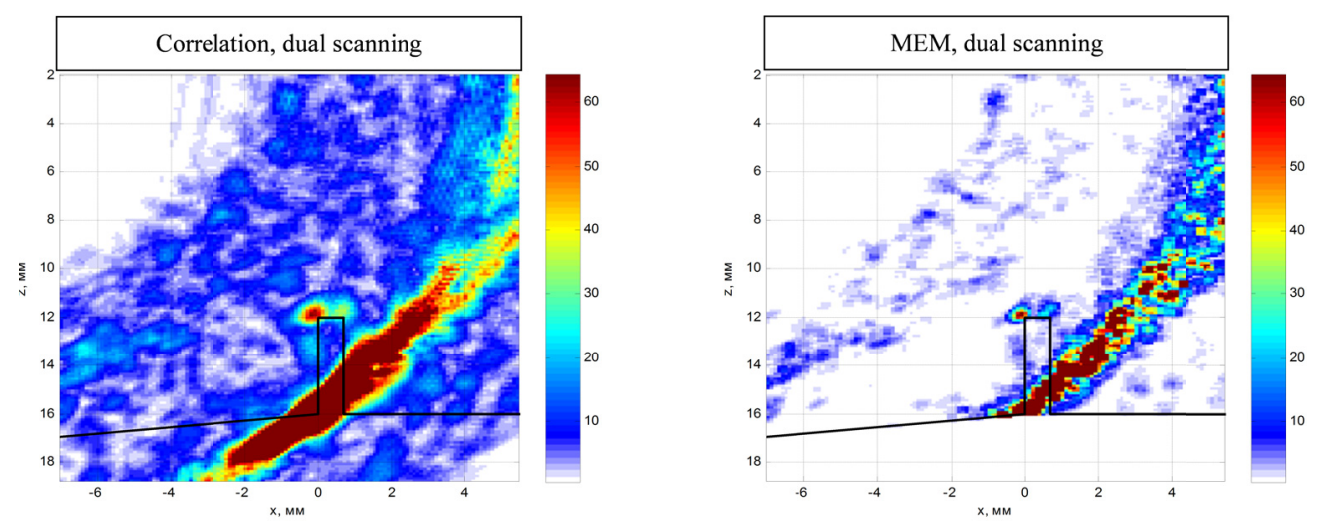

Figure 12. Boundary images of the model of a vertical crack for acoustic T(S)-T(S)

The left of Figure 13 shows an image of the boundary of the crack model restored by correlation method using formula (8) on the left, and the right - by MEM using formula (2) and (9), after 10 iterations at $\alpha=1.5$ и $\mu=10^{-5}$. When calculating matrix $G$ three acoustic schemes T(S)-T(S), T(S)-TB(SS) and TB(SS)-TB(SS) were used. The image of crack model obtained by MEM has a higher resolution, and signal/noise ratio, compared to the image obtained by the correlation method, increased by $12 \mathrm{~dB}$. The amplitude of false glares which arise due to a large distance between the elements of antenna array, significantly decreased. Images in Figure 12 and Figure 13 were obtained from 140 randomly selected echo signals, that is approximately $13.7 \%$ of all 1024 measured echo signals.
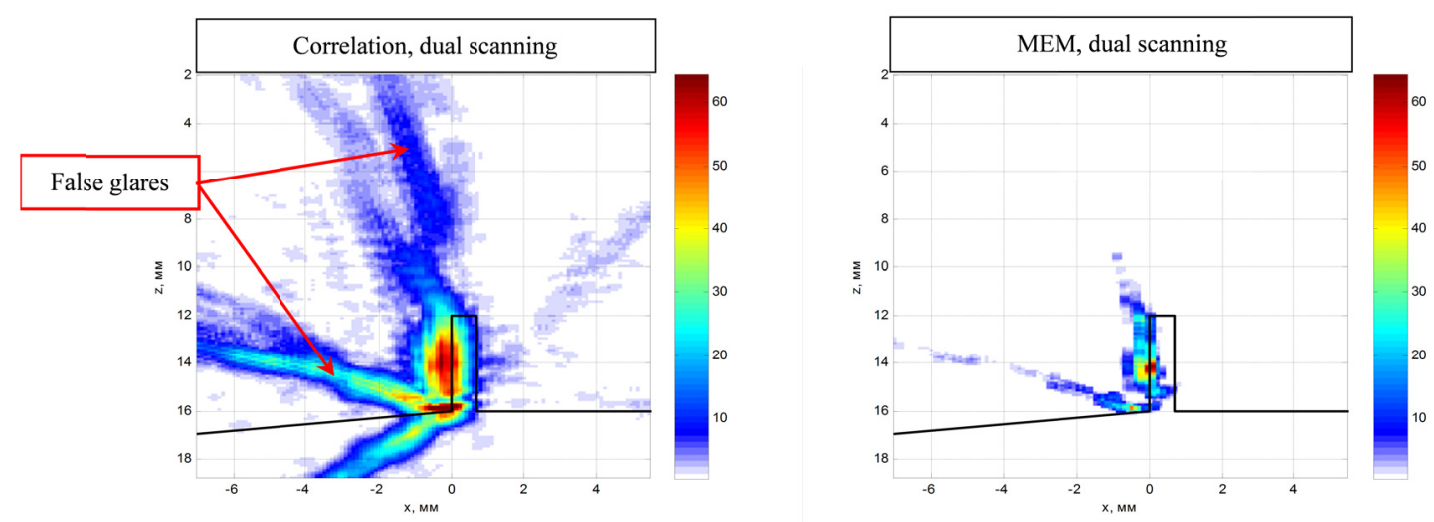

Figure 13. Boundary images of the model of a vertical crack for acoustic schemes T(S)-T(S), T(S)-TB(SS) and TB(SS)-TB(SS)

\subsection{Side drilling hole of $2 \mathrm{~mm}$ diameter at a $12 \mathrm{~mm}$ depth in the specimen with an uneven bottom}

To demonstrate the feasibility of reconstructing the shape of deflectors using two antenna arrays the following simulation experiment was conducted. Figure 14 shows a photograph of a specimen of $h=18$ mm thickness with a model of volumetric defect in the form of the side drilling hole with a diameter of $2 \mathrm{~mm}$ at a depth of 6 $\mathrm{mm}$. For receiving the echo signals in the dual scanning mode there were used two antenna arrays working on 5.0 $\mathrm{MHz}$ operating frequency, consisting of 32 elements with a pitch of $1.0 \mathrm{~mm}$ and mounted on rexsolite prisms of $35^{\circ}$ incline shown schematically in Figure 14. The notion of acoustic channel involves the setting up of 
equipment describing the way of working with the dual scanning by one or two antenna arrays, the value of the amplification gain of the receiver, the parameters of the probe impulse, the temporary aperture of echo signals acquisition and etc. The measurement cycle consists of the emitting and receiving the radiation by antenna array from both the N-side (blue arrow, N-channel) and P-side (green arrow, P-channel), the emitting the ultrasonic impulses by the antenna array from the $\mathrm{N}$-side and receiving by antenna array on $\mathrm{P}$-side (two yellow arrows, NP-channel).

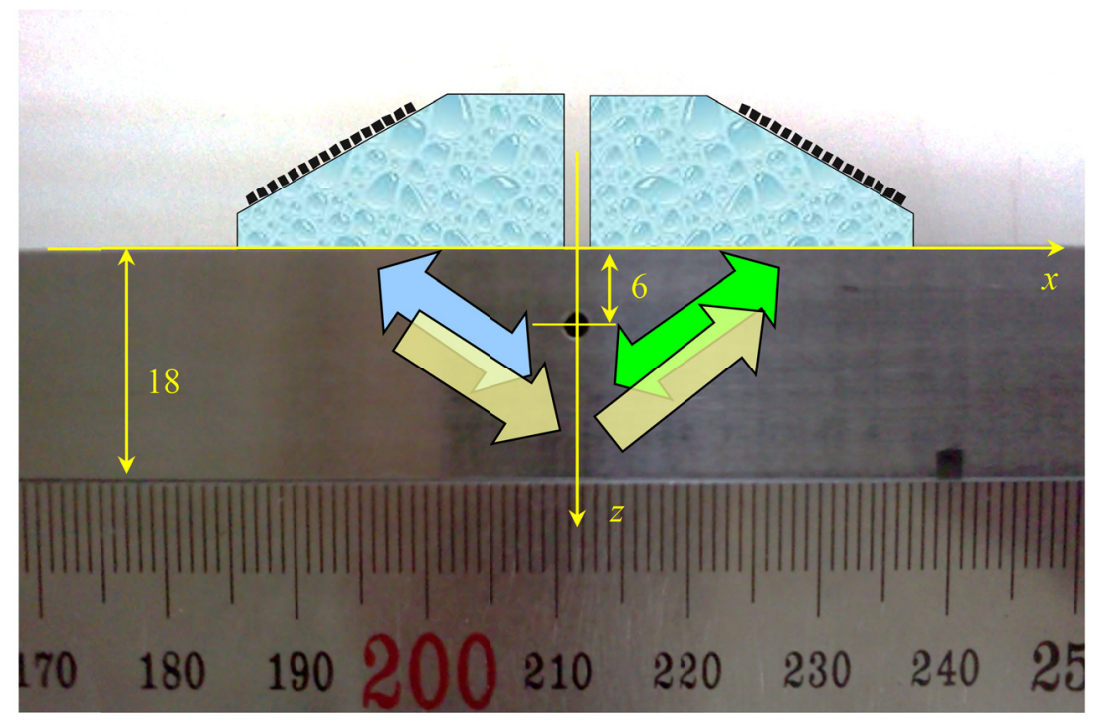

Figure 14. Specimen photo with side drilling hole with a diameter of $2 \mathrm{~mm}$ at a depth of $6 \mathrm{~mm}$

Since the prisms were put almost tightly to one another, three acoustical schemes T(S)-T(S), T(S)-TB(SS) and TB(SS)-TB(SS) were used in course of deflectors images restoring by TS-MC-SAFT method. Figure 15 shows the image obtained by TS-MC-SAFT method from a set of echo signals measured in the mode of double scanning by N-, P- and NP-channel. The left panel of Figure 15 shows the image obtained by coherent addition of all partial images, and the right panel - by adding modules of partial images. The specimen bottom and the boundaries of side drilling hole are masked with black lines. Small amount of angles of the emitted and deflected field caused the effect when a coherent picture of side drilling hole boundaries appeared to be fragmented into several glares. On the right image the contour of boundaries is prescribed without splitting into glares, but with less resolution and with signal/noise ratio by $3 \mathrm{~dB}$ less than the on the left image. To build the image by TS-MC-SAFT method, all 3072 echo signals measured by N-, P- and NP-channel were used.
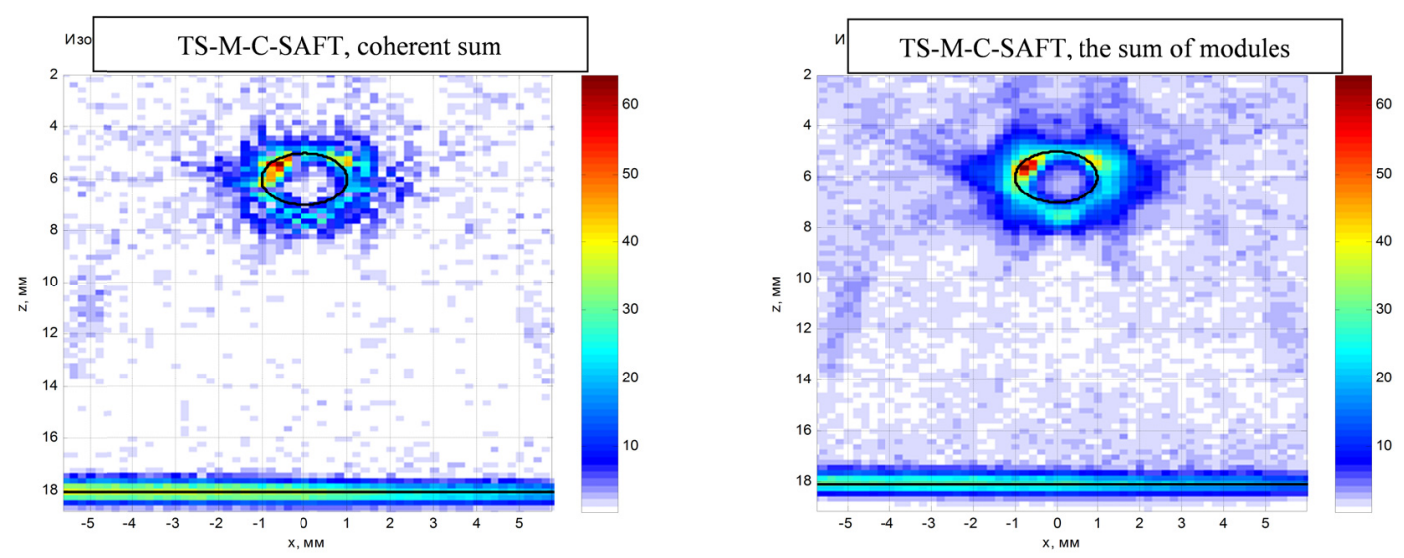

Figure 15. The images of the side drilling hole boundary restored by TS-MC-SAFT method by echo signals measured in double scanning mode by N-, P- and NP-channel 
The resulting image was built by correlation method and MEM in the following way: the images had been restored by N-, P- and NP-channels and then coherently added. The left picture of Figure 16 shows an image of the side drilling hole edge restored by correlative method using formula (8), and the right one - by MEM using formula (2), (9) and after 10 iterations at $\alpha=3$ and $\mu=10^{-5}$ had been made. When calculating matrix $G$, three acoustic schemes $\mathrm{T}(\mathrm{S})-\mathrm{T}(\mathrm{S}), \mathrm{T}(\mathrm{S})-\mathrm{TB}(\mathrm{SS})$ and $\mathrm{TB}(\mathrm{SS})-\mathrm{TB}(\mathrm{SS})$ were used for each channel. A set of 280 echo signals were randomly selected from the total sample, which is about $9 \%$ of all measured echo signals. This caused the fact that the correlation image of the hole border became almost useless for analysis because of the high amplitude of glares. In the same time, the signal/noise ratio of an image obtained by MEM increased by $8 \mathrm{~dB}$, thus allowing for a greater certainty in determining the deflector shape. To reduce the effect of border glare splitting, it is necessary to increase the number of both the angles of coincidence and the angles of reflection from the scatterer by using more acoustic schemes and carrying out the echo signals registration in a triple scanning mode. So, in case of a continuous antenna it is possible to have the directivity diagram of single petal shape, but the main petal of any antenna consisting of a few elements will certainly have numerous dips.
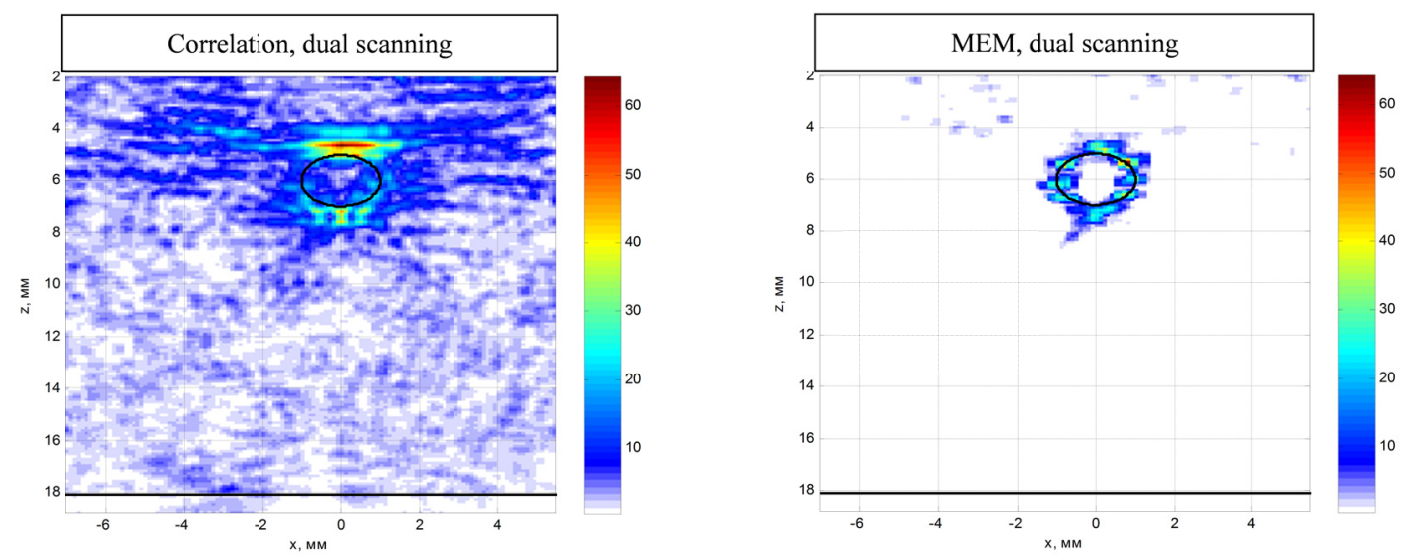

Figure 16. Images of side drilling hole border restored by correlation method (left) and MEM (right) using the echo signals measured in double scanning mode by N-, P-and NP-channel

\subsection{Compressing the complex signals using MEM at high levels of ultrasonic attenuation}

MEM can be used for either simple or complex signals deconvolution. Convolution operation can be written in matrix form as follows

$$
s=G s^{\infty}+n,
$$

where $S$ is a vector-column of $N$ counts length, containing measured echo signal, $G$ is circulant matrix $N \times N$ with the columns being formed by the reference echo signal, $s_{e}(t), s^{\infty}-$ the undistorted function which is to be restored, $n$ is a vector-column of the measurements noise. Using the expression (17) allows to begin seeking for estimation $\hat{S}^{\infty}$ by the maximum entropy method, as was described in section 2 and with accordance with formula (9)

$$
\hat{S}_{\alpha}^{\infty}=\underset{s \in \mathbb{R}^{N}}{\arg \min }\left(\left\|G \hat{S}^{\infty}-s\right\|-\alpha H\left(\hat{S}^{\infty}\right)\right) .
$$

Thus, to deconvolution the signals (17), one can use both the solution of the optimization problem using the formula (18) and calculation of the entropy, its gradient and Hessian by the formula (13). At solving the equation (17), such a deconvolution method should provide the signal estimate $\hat{s}^{\infty}$ restoring with a super resolution.

Practical application of the proposed algorithm for echo signals compression is impossible without demonstrating its resistance to noise. In the model experiment echo signals reflected from $2.0 \mathrm{~mm}$ diameter holes in the standard specimen CO-1 were registered (Figure 17. Measurements were performed with a piezoelectric transducer with resonance frequency $4.0 \mathrm{MHz}$, the pitch of $40^{\circ}$ and a half opening angle of $20^{\circ}$ determined by the level of $10 \%$. The scan area is shown schematically in Figure 17 by several transducer images. To get a simple signal, a bipolar impulse of $200 \mathrm{~ns}$ was sent to the generator input, while to get a complex signal, the transducer is excited by a series of 32 bipolar impulses phase-shifted with accordance to pseudorandom M-sequence. 


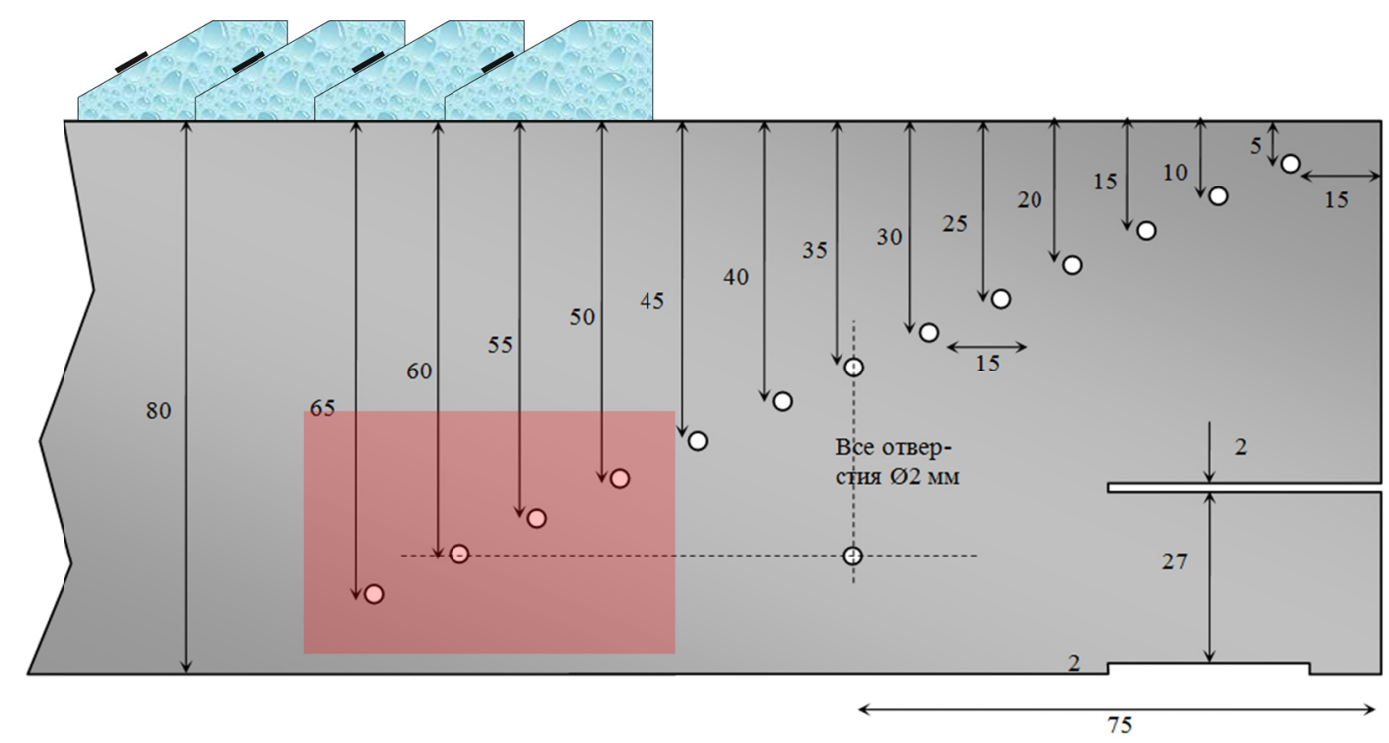

Figure 17. Fragment of the specimen CO-1. ROI is marked by red rectangle

In the left panel of Figure 18 an image of initial echo signals are shown in raster form, also with the result of their compressing by matched filtration and by MEM compression. After MEM compression had been applied, the pulse length became 5 times shorter in comparison with one got by matched filtration compressing, and the level of noise and «side lobes» decreased by more than $40 \mathrm{~dB}$.

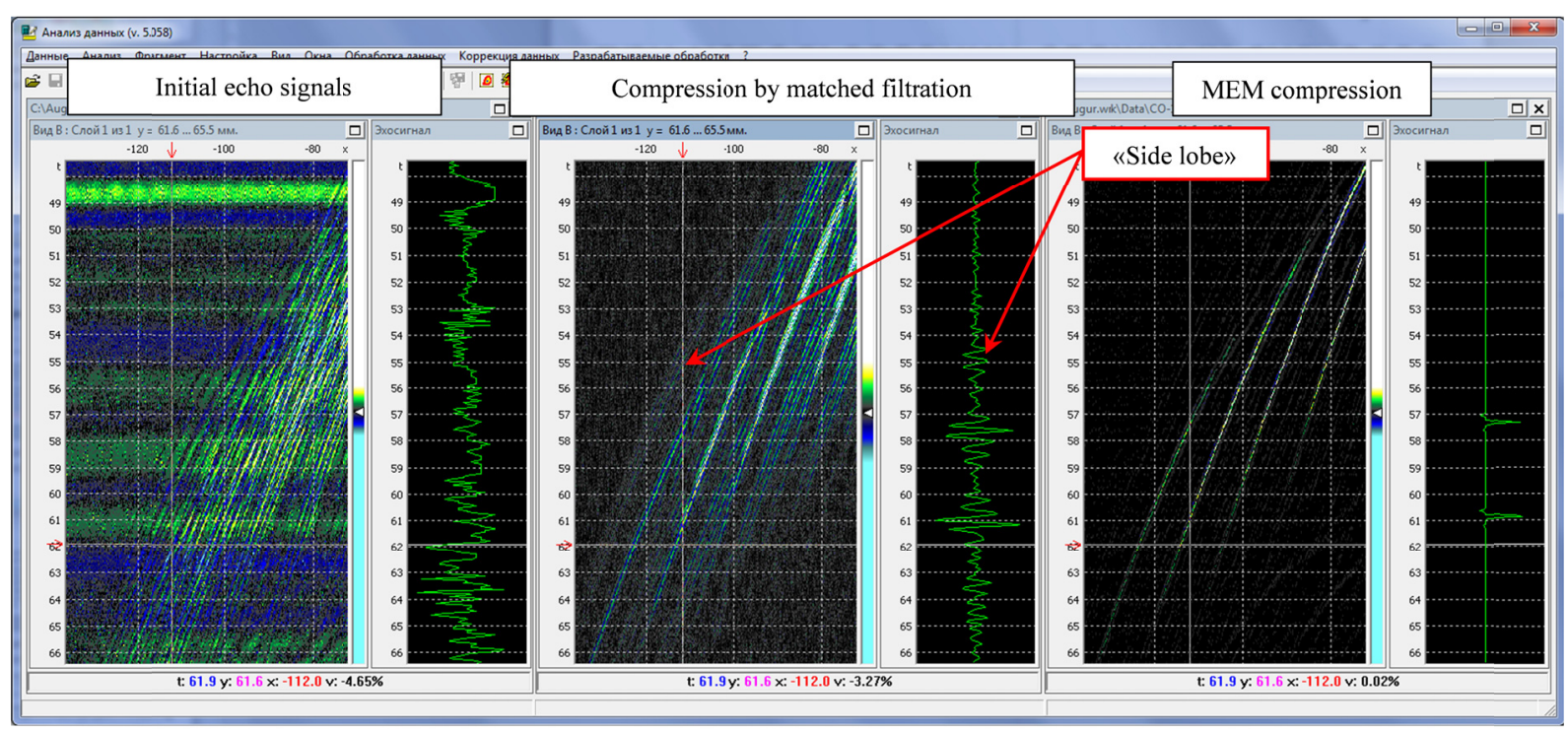

Figure 18. Initial echo signals, the result of their compression by matched filtration and MEM

Left in the top row in Figure 19 shows the result of a simple visualization of side holes images in case of simple pulses radiation, while right - the result of restoring by PSS method and with help of the same echo signals the holes borders images with outlines shown in the figure with black circles. Signal/noise ratio of the images obtained by the PSS method increased by more than $20 \mathrm{~dB}$ if compared to a simple visualization, while the frontal resolution decreased from $25 \mathrm{~mm}$ to $4 \mathrm{~mm}$. The bottom row shows the images of the holes boundaries restored by PSS method with help of echo signals compressed by matched filtering (left) and echo signals compressed by MEM (right). It is seen that after MEM compression had been carried out the level of «side lobes» decreased practically from $-10 \mathrm{~dB}$ to $-50 \mathrm{~dB}$ with respect to the amplitude of the «main» lobe, and beam 
resolution also decreased from $0.9 \mathrm{~mm}$ to $0.2 \mathrm{~mm}$.

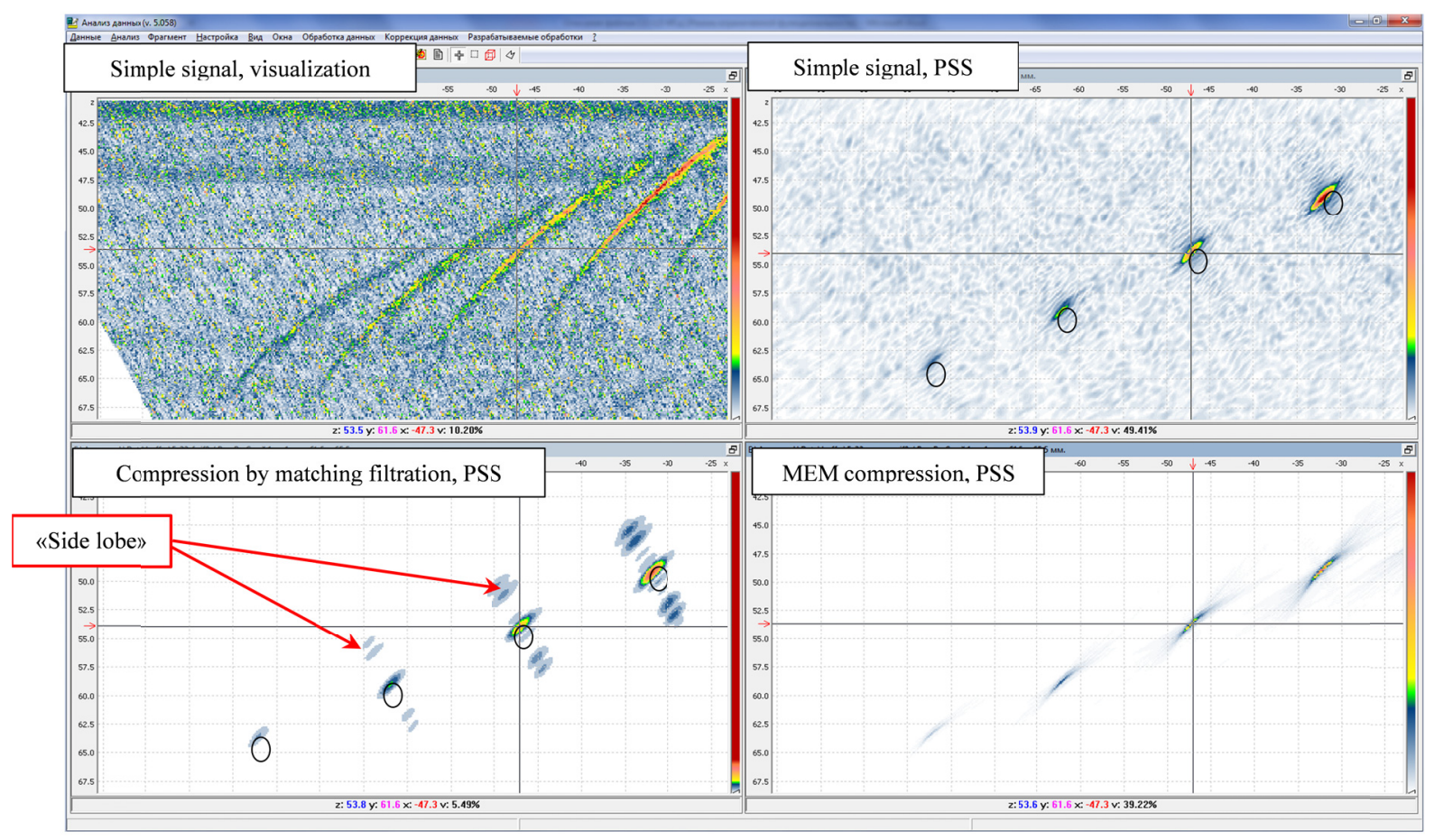

Figure 19. Images restored by the initial echo signals and ones after their compressing by matching filtration and

MEM

\section{Conclusions}

Thus, basing on research findings outlined in this article, we can draw the following conclusions:

1) To restore the deflector images by echo signals it is proposed to use the MEM. Its fundamental differences from the imaging method TS-MC-SAFT are:

a) there is no need in forming a great number of partial images by selected acoustic schemes, and then combine them into one final image - the MEM provides a single image at once, with all mentioned acoustic schemes already taken into account;

b) the image restored by the MEM has low noise level and, in the same time, high longitudinal and frontal resolution;

c) Only about $10 \%$ of echo signals received either in dual or in triple scanning mode are sufficient for restoring an image by the MEM. Lowering of the minimum sufficient number of echo signals should increase the rate of their registration, which is very important in automated ultrasonic testing.

2) Model experiments showed high efficiency of the MEM at restoring of flaw images by echo signals measured by a single-element transducer working in matching mode, and also at the receiving the echo signals in dual and triple scanning mode using antenna arrays.

3) The deflector images restoring in materials featuring high level of structural noise showed the effectiveness of MEM usage.

4) MEM can be used to compress complex signals, which improves the quality of images of flaws in materials with high attenuation. MEM works less reliably with short signals.

5) Application of MEM can significantly distort the amplitudes of deflector glares.

The author expresses gratitude to Vadim Zotov for correction of the English text of the article, which significantly improved the quality of its translation. 


\section{References}

Badalyan V. G, Bazulin E.G. (1988). Digital image restoration of scatterers by the method of projection in the spectral space. Acoust. Journ, 2, 222-231.

Battle, D. J. (1999). Maximum Entropy Regularization Applied To Ultrasonic Image Restoration. University of Sydney.

Baykova, A. T. (2008). Multi-frequency synthesis of images in VLBI based on generalized method of maximum entropy. Astron. Journ., 12, 1059-1071.

Bazulin E. G. (2013). Comparison of systems for ultrasonic nondestructive testing using antenna arrays or phased antenna arrays. Russian Journal of Nondestructive Testing., 7, 51-75. http://dx.doi.org/10.1134/S1061830913070024

Bazulin E. G., Golubev A. S., Kokolev S. A. (2009). Use of an ultrasound antenna array for acquisition of echosignals by dual scan method to get flaw images. Russian Journal of Nondestructive Testing, 2, 18-32.

Bazulin, A. E., \& Bazulin, E. G. (2005). About a possibility of using the maximum entropy method for improving the image quality of scatterers in ultrasonic nondestructive testing. Acoust. Journ., 5, 589-601.

Bazulin, E. G. (1991). Application of the two-dimensional adaptive extrapolation method for improving of the quality of the scatterers image restored by multi-frequency and multi-field-aligned acoustic holograms. Acoust. Journ., 1, 8-16.

Bazulin, E. G. (1993). Getting images of scatterers with longitudinal superresolution by multifrequency digital acoustic holograms in the construction of AR-model of echo signals spectra. Acoust. Journ., 2, 213-222.

Bazulin, E. G. (2010). Application of the maximum entropy method in ultrasonic nondestructive testing to get images of scatterers taking into account the effect of rescattering in one-dimensional case. Acoust. Journ, 1, 103-112.

Bazulin, E. G. (2011). Determining the Flaw Type from Images Obtained by the CSAFT Method with Account for Transformationsof Wave Types upon Reflections of Ultrasonic Pulses from the Irregular Boundaries of a Test Object. Russian Journal of Nondestructive Testing, 1, 39-56. http://dx.doi.org/10.1134/S1061830911010037

Born, M., \& Wolf, E. (1999). Principles of Optics (7th ed.). New York: Cambridge University Press. http://dx.doi.org/10.1017/CBO9781139644181

Box, G. E. P., Jenkins, G. M., \& Reinsel, G. C. (1994). Time series analysis - Forecasting and control (3rd ed.). Prentice Hall. Englewood Cliffs. NJ. USA. http://dx.doi.org/10.1002/9781118619193.

Braconnier D., Okuda S., Dao G. (2009). A Detailed Study of Inspecting Thick Parts Using Large Aperture Phased Arrays and DDF. 7th International Conference on NDE in Relation to Structural Integrity for Nuclear and Pressurized Components. 12-15 May 2009. Yokohama. Japan.

Brekhovskikh, L. M., \& Godin, O. A. (1992). Acoustics of Layered Media II. Springer Series on Wave Phenomena, 10, 395. http://dx.doi.org/10.1007/978-3-662-02776-9

Burov ,V. A., Shmelev, A. A. (2009). Numerical and physical modeling of the process of tomography based on acoustic nonlinear effects of the third order. Acoust. Journ., 4-5, 466-480.

Burov V. A., Prudnikova, I. P. (1999). An iterative algorithm of solving the inverse boundary problem of ultrasound scattering on the cavity in an isotopic solid body. Acoust. Journ., 6, 759-766.

Burov, V. A., Gurinovitch, I. E., Rudenko, O. V., Tagunov, E. Y. (1994). Reconstruction of the spatial distribution of the nonlinearity parameter and the speed of sound in the acoustic nonlinear tomography. Acoust. Journ., 6 , 922-929.

Burov, V. A., Vecherin, S. N., Morozov, S. A., \& Rumyantsev, O. D. (2010). Modeling of accurate solution of the inverse problem of acoustic scattering by functional methods. Acoust. Journ., 4, 516-536.

Chatillon S., Fidahoussen A., Iakovleva E., Calmon P. (2009). Time of flight inverse matching reconstruction of ultrasonic array data exploiting forwards models. NDT in Canada 2009 National Conference.

Danilov V. N., Samokrutov A. A., Lyutkevich A. M. (2003). Theoretical and experimental studies of small aperture rectangular transducers. Control. Diagnostics, 7, 29-33.

Devaney A. J. (2000). Super-resolution processing of multi-static data using time reversal and MUSIC. Department of Electrical and Computer Engineering. Northeastern University. Boston. USA. 
Erhard, A., Lucht, B., Schulz, E., Montag, H. J., Wüstenberg, H., \& Beine, U. (2000). Characterization of defects in austenitic pipe gird welds. The e-Journal of Nondestructive Testing \& Ultrasonics, 5(9), 1435-1434.

Ermolov, I. N., \& Basatskaya, L. V. (1989). On the question of experimental measurement of the diagrams of transducers direction. Russian Journal of Nondestructive Testing, 4, 3-7.

Ermolov, I. N., \& Lange, Y. V. (2004). Ultrasonic testing. In V. V. Klyueva (Ed.), Non-destructive Testing / Directory / Under general (Vol. 3). M.: Mechanical Engineering.

Faur, M., Morisseau, P., \& Poradis, L. (1998). Ultrasonic data inversion for outer surface defects characterization. 7th European conference on nondestructive testing. Copenhagen, 2429-2436.

Francini, E., Höft, T., \& Santosa, F. (2006). An inverse problem in nondestructive evaluation of spot-welds. Inverse Problems, 22, 645-661. http://dx.doi.org/10.1088/0266-5611/22/2/016.

Freiden, B. R. (1972). Restoring with maximum likelihood and maximum entropy. Journal of The Optical Society of America, 62, 511-518. http://dx.doi.org/10.1364/JOSA.62.000511.

Gengembre N. (2003). Pencil method for ultrasonic beam computation. Proc. of the $5^{\text {th }}$ Word Congress on Ultrasonic, Paris.

Gershberg, R. W. (1974). Super resolution through error energy reduction. Opt. acta., 21, 709-720. http://dx.doi.org/10.1080/713818946

Goncharsky, A. V., \& Romanov, S. Y. (2011). About one problem in ultrasound imaging. Computational methods and Programming, 12, 317-320.

Goodman, J. (1968). Introduction to Fourier Optics. New York: McGraw-Hill Book Co.

Gorjunov, A. A., \& Saskovets, A. V. (1989). Inverse scattering problems in acoustics. M. : MSU Publishing house.

Granichin, O. N. (2009). Stochastic Optimization in Informatics. St. Petersburg : St. Petersburg University Publishing house. 2009.

Hall, T. E., Doctor, S. R., Reid, L. D., Littlefield, R. J., \& Gilbert, R. W. (1987). Implementation of a Real-Time Ultrasonic SAFT System for Inspection of Nuclear Reactor Components. In Proceedings of the Fifteenth International Symposium, July 14-6, 1986 (pp. 253-266). Springer US.

Hansen, P. C. (2001). The L-curve and its use in the numerical treatment of inverse problems. In Computational Inverse Problems in Electrocardiology (pp. 119-142). Southampton: WIT Press.

Helstrom, C. W. (1967). Image Restoration by the Method of Least Squares. J. Opt. Soc. Am., 57(3), 297-303. http://dx.doi.org/10.1364/JOSA.57.000297

Holmes, C., Drinkwater, B. W., \& Wilcox, P. D. (2005). Post-processing of the full matrix of ultrasonic transmit-receive array data for non-destructive evaluation. NDT\&E International, 38, 701-711. http://dx.doi.org/10.1016/j.ndteint.2005.04.002

Hore, P. J. (1991). Maximum Entropy and nuclear magnetic resonance. Maximum Entropy in Action (pp. 41-72). Oxford : Clarendon Press.

Kovalev A. V., Kozlov V. N., Samokrutov, A. A., Shevaldykin, V. G., \& Yakovlev, N. N. (1990). Pulse echomethod at the test of concrete. Noise and spatial selection. Russian Journal of Nondestructive Testing, 2, $29-41$.

Kravtsov, Y. A., \& Orlov, Y. I. (1980). Geometrical optics of inhomogeneous media. M.: Nauka. http://dx.doi.org/10.1007/978-3-642-84031-9.

Kullback, S. (19668). Information Theory and Statistics. New York.

Lasaygues, P., \& Lefebvre, J.-P. (1998). Improvement of resolution in ultrasonic reflection tomography. 7th European conference on nondestructive testing. Copenhagen., 3001-3008.

Letova, T. A., \& Panteleev, A. V. (1998). The extremum of functions in examples and problems: Textbook. M. : MAI Publishing house.

Lingvall, F., Olofsson, T., Wennerström, E., \& Stepinski, T. (2004). Optimal linear receive beamformer for ultrasonic imaging in NDT. 16th WCNDT 2004. Aug 30 - Sep 3. 2004. Montreal, Canada.

Maisinger, K., Hobson, M. P., \& Lasenby, A. N. (2004). Maximum-entropy image reconstruction using wavelets. Mon. Not. R. Astron. Soc., 1, 339-354. http://dx.doi.org/j.1365-2966.2004.07216.x. 
Marple, Jr. S. L. (1987). Digital Spectral Analysis with Applications. New York: Prentice-Hall, Inc., Englewood Cliffs. http://dx.doi.org/10.1121/1.398548.

Mayer, K., Markelein, R., Langenberg, K. J., \& Kreutter, T. (1990). Three-dimensional imaging system based on Fourier transformation synthetic aperture focusing technique. Ultrasonics, 28, 241-255. http://dx.doi.org/10.1016/0041-624X(90)90091-2

Morozov, V. A. (2003). Algorithmic foundations of methods for solving ill-posed problems. Computational Mathematics and Programming, 4, 130-141.

Morozov, V. A. (2004). Some general conditions of regularization of ill-posed variational problems. Computational Mathematics and Programming, 5, 31-40.

Mottershead, C. T. (1996). Maximum Entropy Tomography. Maximum Entropy and Bayesian Methods, 425-436. http://dx.doi.org/10.1007/978-94-011-5430-7_54.

Novikov R. G., Henkin G. M. (1987). $\bar{\partial}$-Equation in the multidimensional inverse scattering problem. Advances of Mathematical Sciences, 3, 93-152.

Novikov, R. G. (1986). Restoring of the two-dimensional Schrödinger operator by the scattering amplitude at a fixed energy. Functional analysis and its applications, 3, 90-91.

Olympus NDT. (2007). Advances in Phased Array ultrasonic technology applications. Retrieved from http://www.olympus-ims.com/en/books/

Osetrov, A. V. (1991). The theory of space-time diffraction tomography at the scanning of a single transceiver transmitter by the plane. Acoust. Journ, 3, 528-534.

Papoulis, A., \& Chamras, D. (1979). Detection of hidden periodicties by adatpive extrapolation. IEEE Trans. Acoust. speech signal proc., 25, 492-500.

Pickalov, V. V., \& Kazantsev, D. I. (2008). Properties of regularized Gerchberg-Papoulis algorithm in fan-beam tomography problem. Computational Technology, 6, 121-133.

Samokrutov, A. A., \& Shevaldykin, V. G. (2011). The possibility of assessing the nature of metal flaws by ultrasound tomigraph with digital focusing of antenna array. Control. Diagnostics, 10, 63-70.

Shannon, C. E. (1984). A mathematical theory of communication. Bell Systems Technical Journal, 27, 379-423. http://dx.doi.org/j.1538-7305.1948.tb01338.x

Tikhonov, A. N., \& Arsenin, V. Y. (1986). On the solution of ill-posed problems (3rd ed. revised). M.: Nauka.

Valyashko, G. M., \& Strakhov, V. N. (1981). Algorithm of adaptive regularization of linear ill-posed problems. Reports of the USSR Academy of Sciences, 3, 100-113.

Van Kempen, G. M. P., \& Van Vliet, L. J. (2000). The influence of the regularization parameter and the first estimate on the performance of Tikhonov regularized non-linear image restoration algorithms. Journal of Microscopy., 198, 63-75.

Vasilenko, G. I. (1986). Taratorin A.M. Image Restoration. M. : Radio and communication.

Wan, S., Balasundar, I. R., \& Mandayam, A. (2003). Srinivasan Robust Deconvolution of High-Frequency Ultrasound Images Using Higher-Order Spectral Analysis and Wavelets. IEEE Transactions on Ultrasonic: Ferroelectrics, and Frequency Control, 50(10), 1286-1295.

Wernecke, S. J., \& D`Addario, L. R. (1977). Maximum Entropy image reconstruction. IEEE Transactions on Computers, 26, 351-364. http://dx.doi.org/10.1109/TC.1977.1674845.

\section{Copyrights}

Copyright for this article is retained by the author(s), with first publication rights granted to the journal.

This is an open-access article distributed under the terms and conditions of the Creative Commons Attribution license (http://creativecommons.org/licenses/by/3.0/). 